\title{
FIRST-ORDER METHODS FOR GENERALIZED OPTIMAL CONTROL PROBLEMS FOR SYSTEMS WITH DISTRIBUTED PARAMETERS
}

\author{
S. V. Denisov, V. V. Semenov \\ Faculty of Computer Science and Cybernetics, Taras Shevchenko National University of Kyiv, \\ Kyiv, Ukraine, E-mail: \{sireukr, volodya.semenov\}@gmail.com
}

\section{МЕТОДИ ПЕРШОГО ПОРЯДКУ ДЛЯ ЗАДАЧ УЗАГАЛЬНЕНОГО ОПТИМАЛЬНОГО КЕРУВАННЯ СИСТЕМАМИ З РОЗПОДІЛЕНИМИ ПАРАМЕТРАМИ}

\author{
С. В. ДЕнисов, В. В. Семенов
}

Факультет комп'ютерних наук та кібернетики, Київський національний університет імені Тараса Шевченка, Київ, Україна, E-mail: \{sireukr, volodya.semenov\}@gmail.com

\begin{abstract}
The problems of optimization of linear distributed systems with generalized control and first-order methods for their solution are considered. The main focus is on proving the convergence of methods. It is assumed that the operator describing the model satisfies a priori estimates in negative norms. For control problems with convex and preconvex admissible sets, the convergence of several first-order algorithms with errors in iterative subproblems is proved. KEYWORDS: optimal control, distributed parameters system, generalized control, first-order method, convergence.
\end{abstract}

\begin{abstract}
АнотАція. Розглядаються задачі оптимізації лінійних розподілених систем з узагальненим керуванням та методи першого порядку їх розв'язання. Основну увагу приділено обгрунтовуванню збіжності методів. Припускається, що оператор, який описує модель, задовольняє апріорні оцінки в негативних нормах. Для задач керування з опуклими та передопуклими допустимими множинами доведено збіжність декількох алгоритмів першого порядку із похибками в ітераційних підзадачах.

КлЮчові словА: оптимальне керування, система з розподіленими параметрами, узагальнене керування, метод першого порядку, збіжність.
\end{abstract}

\section{ВСТУП}

У роботі розглянуті та обгрунтовувані основні чисельні методи оптимізації лінійних розподілених систем з узагальненим керуванням. Ми припускали, що оператор, який описує модель, задовольняє апріорні оцінки в негативних нормах. Зауважимо, що з використанням теорії оснащених 
гільбертових просторів та методу апріорних оцінок в негативних нормах розглядати задачі імпульсного керування лінійними системами з розподіленими параметрами запропонував С. І. Ляшко [1]. Це дозволило створити загальну теорію сингулярного (узагальненого) оптимального керування лінійними системами $[2,3]$ та розв'язати чимало питань щодо існування оптимальних керувань, керованості [4], побудови необхідних умов оптимальності та чисельних методів оптимізації.

\section{1. ПОСТАНОВКА ЗАДАЧ. ГЛАДКІСТЬ ФУНКЦІОНАЛА ЯКОСТІ}

При дослідженні задач узагальненого оптимального керування системами з розподіленими параметрами будемо використовувати підхід робіт $[3,5]$.

Нехай $\mathcal{L}$ - лінійний диференціальний оператор з частинними похідними, який діє у просторі $L_{2}(Q)\left(Q=(0, T) \times \Omega \subseteq \mathbb{R}^{n+1}-\right.$ регулярна область $)$ з областю визначення $D(\mathcal{L})$, що складається з гладких в $\bar{Q}$ функцій, які задовольняють граничні умови (гр). Формально спряжений оператор позначимо $\mathcal{L}^{+}$, а його область визначення $-D\left(\mathcal{L}^{+}\right)-$множина гладких в $\bar{Q}$ функцій, які задовольняють спряжені граничні умови $\left(\right.$ гр $\left.^{+}\right)$. Припускаємо, що $D(\mathcal{L})$ і $D\left(\mathcal{L}^{+}\right)$щільні в $L_{2}(Q)$.

Нехай відносно $L_{2}(Q)$ побудовано ланцюжки гільбертових оснащень [6]

$$
W \subseteq H \subseteq L_{2}(Q) \subseteq H^{-} \subseteq W^{-}, W_{+} \subseteq H_{+} \subseteq L_{2}(Q) \subseteq H_{+}^{-} \subseteq W_{+}^{-},
$$

де $W, H\left(W_{+}, H_{+}\right)$- поповнення $D(\mathcal{L})\left(D\left(\mathcal{L}^{+}\right)\right)$за позитивними нормами $\|\cdot\|_{W},\|\cdot\|_{H}\left(\|\cdot\|_{W_{+}},\|\cdot\|_{H_{+}}\right)$(як правило, це норми відповідних просторів Соболева); $W^{-}, H^{-}\left(W_{+}^{-}, H_{+}^{-}\right)-$відповідні негативні простори.

Припустимо, що для $\mathcal{L}$ і $\mathcal{L}^{+}$справедливі апріорні оцінки в негативних нормах

$$
\begin{gathered}
c_{1}\|y\|_{H} \leq\|\mathcal{L} y\|_{W_{+}^{-}} \leq c_{2}\|y\|_{W} \quad \forall y \in D(\mathcal{L}), \\
c_{1}\|p\|_{H_{+}} \leq\left\|\mathcal{L}^{+} p\right\|_{W^{-}} \leq c_{2}\|p\|_{W_{+}} \quad \forall p \in D\left(\mathcal{L}^{+}\right),
\end{gathered}
$$

де $c_{1}, c_{2}$ - додатні константи, що не залежать від функцій $y, p$.

Із правих частин оцінок випливає, що $\mathcal{L}, \mathcal{L}^{+}$можна розширити за неперервністю до неперервно діючих $W \rightarrow W_{+}^{-}, W_{+} \rightarrow W^{-}$операторів. Для розширених операторів збережемо старі позначення. Далі, говорячи про оператори $\mathcal{L}$ і $\mathcal{L}^{+}$, будемо розуміти їх розширення. Оцінки в негативних нормах залишаються справедливими при довільних $y \in W, p \in W_{+}$. Для $\mathcal{L}, \mathcal{L}^{+}$виконується тотожність $\langle\mathcal{L} y, p\rangle_{+}=\left\langle y, \mathcal{L}^{+} p\right\rangle$, де $\langle\cdot, \cdot\rangle_{+},\langle\cdot, \cdot\rangle-$ канонічні білінійні форми, побудовані на просторах $W_{+}^{-}, W_{+}$i $W, W^{-}$. За допомогою оцінок

$$
c_{1}\|y\|_{H} \leq\|\mathcal{L} y\|_{W_{+}^{-}} \forall y \in W, \quad c_{1}\|p\|_{H_{+}} \leq\left\|\mathcal{L}^{+} p\right\|_{W^{-}} \quad \forall p \in W_{+},
$$

доводиться існування та єдиність розв'язків з просторів $W, W_{+}$рівнянь

$$
\begin{gathered}
\mathcal{L} y=f, \quad f \in H_{+}^{-}, \\
\mathcal{L}^{+} p=g, \quad g \in H^{-},
\end{gathered}
$$


причому справедливі оцінки

$$
\|y\|_{W} \leq c\|f\|_{H_{+}^{-}},\|p\|_{W_{+}} \leq c\|g\|_{H^{-}} .
$$

Будемо також розглядати розв'язки з більш широких класів.

Узагальненим розв'язком рівняння (1) з $f \in W_{+}^{-}$називаємо елемент $y \in$ $H$ такий, що $\left[y, \mathcal{L}^{+} p\right]=\langle f, p\rangle_{+} \forall p \in W_{+}: \mathcal{L}^{+} v \in H^{-}$, де $[\cdot, \cdot]-$ канонічна білінійна форма, побудована на просторах $H, H^{-}$.

Аналогічно, узагальненим розв'язком спряженого рівняння (2) із правою частиною $g \in W^{-}$називаємо елемент $p \in H_{+}$такий, що $[\mathcal{L} y, p]_{+}=\langle y, g\rangle$ $\forall y \in W: \mathcal{L} y \in H_{+}^{-}$, де $[\cdot, \cdot]_{+}: H_{+}^{-} \times H_{+} \rightarrow \mathbb{R}-$ канонічна білінійна форма.

Відомо, що для довільних елементів $f \in W_{+}^{-}$i $g \in W^{-}$існують єдині узагальнені розв'язки рівняння (1) і спряженого рівняння (2), причому справедливі оцінки

$$
\|y\|_{H} \leq c\|f\|_{W_{+}^{-}}, \quad\|p\|_{H_{+}} \leq c\|g\|_{W^{-}} .
$$

Зауваження 1. Нехай $y \in H \cap W-$ узагальнений розв'язок (1) із правою частиною $f \in W_{+}^{-}$, тоді $y-$ розв'язок рівняння $\mathcal{L} y=f$. Для $f \in R(\mathcal{L})$ узагальнений розв'язок $y$ також задовольняє рівнянню $\mathcal{L} y=f$.

Нехай $\left(V,\|\cdot\|_{V}\right)$ - банахів простір керувань, $F: V \rightarrow W_{+}^{-}$- оператор, що задає вплив на систему. Для керування $u \in V$ стан $y=y(u) \in H$ системи визначається як узагальнений розв'язок рівняння $\mathcal{L} y=F(u)$. Нехай $\Phi: H \times V \rightarrow \mathbb{R}$ - заданий функціонал. Розглянемо задачу оптимального керування

$$
\begin{gathered}
J(u)=\Phi(y(u), u) \rightarrow \inf _{u \in U}, \\
\mathcal{L} y(u)=F(u), u \in U,
\end{gathered}
$$

де $U$ - підмножина допустимих керувань із простору керувань $V$.

Використовуючи результати про узагальнену розв'язність (1), міркування компактності-неперервності, легко довести, що задача (3), (4) має непорожню $\sigma\left(V, V^{*}\right)$-компактну множину розв'язків при виконанні умов:

1) функціонал $\Phi: H \times V \rightarrow \mathbb{R}$ секвенційно напівнеперервний знизу у слабких топологіях просторів $H$ та $V$;

2) оператор $F: V \rightarrow W_{+}^{-}$секвенційно слабко неперервний;

3) множина $U \subseteq D(F)$ компактна в топології $\sigma\left(V, V^{*}\right)$.

Зауваження 2. Сформульованим умовам задовольняють: функціонали вигляду $\Phi(y, u)=\alpha_{0}\|y\|_{H}^{\beta_{0}}+\alpha_{1}\|u\|_{V}^{\beta_{1}}\left(\alpha_{0}, \alpha_{1}, \beta_{0}, \beta_{1}>0\right)$; замкнені опуклі та обмежені підмножини $U$ рефлексивних банахових просторів $\left(V,\|\cdot\|_{V}\right)$; імпульсні, точкові, рухомі та інші узагальнені керуючі впливи $F(\cdot)$ [3].

Зауваження 3. Якщо $J(\cdot)$ задовольняє умову коерцитивності: $J\left(u_{k}\right) \rightarrow$ $+\infty$ при $\left\|u_{k}\right\|_{V} \rightarrow+\infty$, то $\sigma\left(V, V^{*}\right)$-компактна множина оптимальних керувань існує при необмеженій $\sigma\left(V, V^{*}\right)$-замкненій множині $U$ рефлексивного простору $\left(V,\|\cdot\|_{V}\right)$. 
Зауваження 4. Ураховуючи нелінійність оператора $F: V \rightarrow W_{+}^{-}$, функціонал якості $J(\cdot)$ може бути неопуклим і при квадратичному або лінійному функціоналі $\Phi: H \times V \rightarrow \mathbb{R}$, а (3), (4) - багатоекстремальною задачею.

Приділимо увагу диференціальним властивостям функціонала якості $J(\cdot)$.

Теорема 1. Нехай оператор $F: V \rightarrow W_{+}^{-}$y точиi $u \in V$ мае nохідну Фреше $F^{\prime}(u) \in L\left(V, W_{+}^{-}\right)$; функиіонал $\Phi: H \times V \rightarrow \mathbb{R}$ має у точиі $(y(u), u) \in H \times V$ похідну Фреше $\left(\Phi_{1}^{\prime}(y(u), u) \in H^{-}, \Phi_{2}^{\prime}(y(u), u) \in V^{*}\right.$ - відповідні частинні похідні Фреше). Тоді функиіонал якості $J: V \rightarrow \mathbb{R}$ диференчійовний за Фреше у точиі $u \in V$ i його похідна обчислюеться за формулою

$$
\left\langle J^{\prime}(u), h\right\rangle_{V^{*}, V}=\left\langle\left(F^{\prime}(u)\right)^{*} p+\Phi_{2}^{\prime}(y(u), u), h\right\rangle_{V^{*}, V} \quad \forall h \in V,
$$

де $p=p(u) \in W_{+}-$спряжсений стан - розв'язок рівняння

$$
\mathcal{L}^{+} p=\Phi_{1}^{\prime}(y(u), u) .
$$

Доведення. Знайдемо в $u \in V$ лінійну частину приросту $\Delta J(u ; h)=J(u+h)-$ $J(u)$ функціонала якості. Маємо

$$
\begin{aligned}
\Delta J(u ; h)= & \Phi(y(u+h), u+h)-\Phi(y(u), u)= \\
& =\left[y(u+h)-y(u), \Phi_{1}^{\prime}(y(u), u)\right]+ \\
+ & \left\langle\Phi_{2}^{\prime}(y(u), u), h\right\rangle_{V^{*}, V}+o\left(\|y(u+h)-y(u)\|_{H}\right)+o\left(\|h\|_{V}\right) .
\end{aligned}
$$

Введемо спряжений стан $p=p(u) \in W_{+}$як розв'язок рівняння

$$
\mathcal{L}^{+} p=\Phi_{1}^{\prime}(y(u), u) .
$$

Тоді можемо записати

$$
\begin{aligned}
& {\left[y(u+h)-y(u), \Phi_{1}^{\prime}(y(u), u)\right]=} \\
& =\left[y(u+h)-y(u), \mathcal{L}^{+} p\right]=\langle F(u+h)-F(u), p\rangle_{+}= \\
& \quad=\left\langle F^{\prime}(u) h, p\right\rangle_{+}+o\left(\|h\|_{V}\right)=\left\langle\left(F^{\prime}(u)\right)^{*} p, h\right\rangle_{V^{*}, V}+o\left(\|h\|_{V}\right) .
\end{aligned}
$$

Оскільки

$$
\|y(u+h)-y(u)\|_{H} \leq c\|F(u+h)-F(u)\|_{W_{+}^{-}}=O\left(\|h\|_{V}\right),
$$

то

$$
\Delta J(u ; h)=\left\langle\left(F^{\prime}(u)\right)^{*} p, h\right\rangle_{V^{*}, V}+\left\langle\Phi_{2}^{\prime}(y(u), u), h\right\rangle_{V^{*}, V}+o\left(\|h\|_{V}\right) .
$$

Функціонал $J: V \rightarrow \mathbb{R}$ диференційовний за Фреше в точці $u \in V$ і має місце зображення (5).

Теорема 2. Нехай на обмеженій опуклій множині $U \subseteq V$ оператор $u \mapsto$ $F^{\prime}(u)$ задовольняе умову Гельдера з показником $\gamma \in(0,1]$; оператори $(y, u) \mapsto$ $\Phi_{1}^{\prime}(y, u) i(y, u) \mapsto \Phi_{2}^{\prime}(y, u)$ задовольняють на обмежених підмножсинах простору $H \times V$ умову Гелъдера з показником $\gamma \in(0,1]$. Тоді похідна Фреше $J^{\prime}(\cdot)$ задовольняе на множині $U$ умову Гельдера з показником $\gamma$. 
Доведення. Нехай $u_{1}, u_{2}$ - довільні точки з множини $U$. Розглянемо

$$
\begin{aligned}
& \left\|J^{\prime}\left(h_{1}\right)-J^{\prime}\left(h_{2}\right)\right\|_{V^{*}}= \\
& \begin{aligned}
&=\left\|\left(F^{\prime}\left(u_{1}\right)\right)^{*} p_{1}+\Phi_{2}^{\prime}\left(y\left(u_{1}\right), u_{1}\right)-\left(F^{\prime}\left(u_{2}\right)\right)^{*} p_{2}-\Phi_{2}^{\prime}\left(y\left(u_{2}\right), u_{2}\right)\right\|_{V^{*}} \leq \\
& \leq\left\|F^{\prime}\left(u_{1}\right)\right\|\left\|p_{1}-p_{2}\right\|_{W_{+}}+\left\|F^{\prime}\left(u_{1}\right)-F^{\prime}\left(u_{2}\right)\right\|\left\|p_{2}\right\|_{W_{+}}+ \\
&+\left\|\Phi_{2}^{\prime}\left(y\left(u_{1}\right), u_{1}\right)-\Phi_{2}^{\prime}\left(y\left(u_{2}\right), u_{2}\right)\right\|_{V^{*}},
\end{aligned}
\end{aligned}
$$

де $p_{1} \in W_{+}, p_{2} \in W_{+}$- розв'язки рівнянь

$$
\mathcal{L}^{+} p_{1}=\Phi_{1}^{\prime}\left(y\left(u_{1}\right), u_{1}\right), \mathcal{L}^{+} p_{2}=\Phi_{1}^{\prime}\left(y\left(u_{2}\right), u_{2}\right) .
$$

Оскільки оператор $u \mapsto F^{\prime}(u)$ задовольняє умову Гельдера на обмеженій опуклій множині, то він і оператор $u \mapsto F(u)$ є обмеженими. Робимо висновок, що множина $\{y(u) \in H: u \in U\}$ обмежена у просторі $H$ і оператори $\Phi_{1}^{\prime}, \Phi_{2}^{\prime}$ - гельдерові на множині $\{(y(u), u): u \in U\} \subseteq H \times V$. Отже, маємо

$$
\begin{aligned}
\| J^{\prime}\left(h_{1}\right)-J^{\prime}\left(h_{2}\right) & \left\|_{V^{*}} \leq C_{0}\right\| \Phi_{1}^{\prime}\left(y\left(u_{1}\right), u_{1}\right)-\Phi_{1}^{\prime}\left(y\left(u_{2}\right), u_{2}\right) \|_{H^{-}}+ \\
& +C_{1}\left\|u_{1}-u_{2}\right\|_{V}^{\gamma}\left\|\Phi_{1}^{\prime}\left(y\left(u_{2}\right), u_{2}\right)\right\|_{H^{-}}+ \\
+ & C_{3}\left(\left\|y\left(u_{1}\right)-y\left(u_{2}\right)\right\|_{H}+\left\|u_{1}-u_{2}\right\|_{V}\right)^{\gamma} \leq \\
\leq & C_{4}\left\|u_{1}-u_{2}\right\|_{V}^{\gamma}+C_{5}\left(\left\|y\left(u_{1}\right)-y\left(u_{2}\right)\right\|_{H}+\left\|u_{1}-u_{2}\right\|_{V}\right)^{\gamma} .
\end{aligned}
$$

Залишилось скористатись нерівністю

$$
\begin{aligned}
& \left\|y\left(u_{1}\right)-y\left(u_{2}\right)\right\|_{H} \leq c\left\|F\left(u_{1}\right)-F\left(u_{2}\right)\right\|_{W_{+}^{-}} \leq \\
& \quad \leq c \sup _{\theta \in[0,1]}\left\|F^{\prime}\left(u_{1}+\theta\left(u_{2}-u_{1}\right)\right)\right\|\left\|u_{1}-u_{2}\right\|_{V} \leq C_{6}\left\|u_{1}-u_{2}\right\|_{V} .
\end{aligned}
$$

Звідси випливає потрібне.

\section{2. ЗБІЖНІСТЬ МОДЕЛЕЙ АЛГОРИТМІВ УЗАГАЛЬНЕНОЇ ОПТИМІЗАЦІЇ ЛІНІЙНИХ СИСТЕМ}

Перейдемо до дослідження алгоритмів наближеного розв'язання описаних вище задач узагальненого оптимального керування лінійними системами з розподіленими параметрами.

Розглянемо задачу оптимального керування

$$
\begin{gathered}
J(u)=\Phi(y(u), u) \rightarrow \inf _{u \in U}, \\
\mathcal{L} y(u)=F(u), u \in U,
\end{gathered}
$$

де $F: V \rightarrow W_{+}^{-}, U-$ множина допустимих керувань із гільбертового простору керувань $V$. Припускається, що

множина $U-$ компактна в сильній топології та опукла.

У задачах оптимального керування системами з розподіленими параметрами сильна компактність, як правило, відсутня, але за рахунок параметризації (регуляризації) керування [3] задачу можна апроксимувати так, щоб мала місце сильна компактність. 
Далі, якщо не вказано інше, будемо вважати, що виконані такі припущення про гладкість даних задачі (6), (7):

1) на множині $U \subseteq V$ оператор $u \mapsto F^{\prime}(u)$ задовольняє умову Гельдера з показником $\gamma \in(0,1]$;

2) оператори $(y, u) \mapsto \Phi_{1}^{\prime}(y, u)$ і $(y, u) \mapsto \Phi_{2}^{\prime}(y, u)$ задовольняють на обмежених підмножинах простору $H \times V$ умову Гельдера з показником $\gamma \in(0,1]$.

Із теореми 2 випливає, що тоді похідна Фреше $J^{\prime}(\cdot)$ задовольняє на $U$ умову Гельдера з показником $\gamma$.

Структура алгоритмів наступна: будується послідовність керувань

$$
u_{n+1}=u_{n}+\rho_{n}\left(\bar{u}_{n}-u_{n}\right),
$$

що задовольняють умову $u_{n} \in U$, а керування $\bar{u}_{n} \in$ наближеним розв'язком деякої допоміжної екстремальної задачі.

Ефективні на практиці алгоритми часто мають напівемпіричний характер. Деякі з них містять не до кінця формалізовані етапи, що часто робить неможливим доведення їх збіжності у тому виді, в якому вони сформульовані. Внаслідок цього можна стверджувати, що, як правило, математики в даній галузі займаються побудовою та дослідженням моделей ітераційних алгоритмів оптимізації - більш простих алгоритмів, що зберігають основні риси методів практичних розрахунків (див. [7-11]).

Для доведення збіжності алгоритмів будемо використовувати метод, розвинутий у роботах київської школи негладкої та стохастичної оптимізації $[8,12,13]$. Ця методика у певній мірі є незалежною від конкретної структури алгоритму та складається з перевірки набору стандартних умов, виконання яких для певного алгоритму гарантує його збіжність. Цей підхід дозволяє для цілого класу алгоритмів провести значну частину доведення збіжності в рамках доведення загальних теорем збіжності, завдяки чому викладення результатів спрощується.

Розглянемо наступну абстрактну задачу:

задано підмножину $X^{*}$ повного метричного простору $(X, \rho)$; знайти точку підмножини $X^{*}$.

У задачах оптимізації для опису множини $X^{*}$, як правило, використовують необхідні умови оптимальності.

Під ітераційним алгоритмом розв'язання абстрактної задачі розуміємо певне правило побудови послідовності точок $\left(x_{n}\right)$ метричного простору $X$. Алгоритм вважаємо збіжним, якщо всі граничні точки послідовності $\left(x_{n}\right)$ належать множині $X^{*}$.

Теорема 3. Припустимо, що:

1) існуе компакт $K \subseteq X: x_{n} \in K \forall n \in \mathbb{N}$;

2) для довільної збіжної підпослідовності $\left(x_{n_{k}}\right)$ виконано умови:

а) якщо $\lim _{k \rightarrow \infty} x_{n_{k}}=x^{\prime} \in X^{*}$, mо $\rho\left(x_{n_{k}+1}, x_{n_{k}}\right) \underset{k \rightarrow \infty}{\longrightarrow} 0$; 
b) якщо $\lim _{k \rightarrow \infty} x_{n_{k}}=x^{\prime \prime} \notin X^{*}$, mo $\exists \delta_{0}>0: \forall \delta \in\left(0, \delta_{0}\right] \tau_{k}<$ $+\infty$, de

$$
\tau_{k}=\min _{n>n_{k}}\left\{n: \rho\left(x_{n}, x_{n_{k}}\right)>\delta\right\}
$$

3) існуе неперервна на множині граничних точок послідовності $\left(x_{n}\right)$ функиія $W: X \rightarrow \mathbb{R}$ така, що для довільної підпослідовності з 2 b)

$$
\limsup _{k \rightarrow \infty} W\left(x_{\tau_{k}}\right)<\lim _{k \rightarrow \infty} W\left(x_{n_{k}}\right) ;
$$

4) множина $W^{*}=W\left(X^{*}\right)=\left\{W(x): x \in X^{*}\right\} \subseteq \mathbb{R}$ мае скрізь щільне доповнення.

Тоді послідовність $\left(W\left(x_{n}\right)\right)$ мае граничо та всі граничні точки послідовності $\left(x_{n}\right)$ утворюють зв'язну компактну підмножину $X^{*}$.

Зауваження 5. Якщо опустити умови 2 а) і 4, то можна стверджувати лише, що існуть граничні точки послідовності $\left(x_{n}\right)$, які належать множині $X^{*}$. Відмітимо, що у формулюванні наведеної теореми відтворено схему доведення збіжності алгоритму від супротивного, тому деякі умови теореми без контексту доведення від супротивного є психологічно незручними.

При формулюванні алгоритмів будемо вважати, що із заданою (в окремих випадках довільною) точністю може бути розв'язана задача

$$
\left\langle u^{*}, u\right\rangle_{V^{*}, V} \rightarrow \inf _{u \in U}, u^{*} \in V^{*}
$$

або задача

$$
\left\langle u^{*}, u\right\rangle_{V^{*}, V}+\frac{1}{2}\|u\|_{V}^{2} \rightarrow \inf _{u \in U}, u^{*} \in V^{*} .
$$

Множини $U$, що мають такі властивості, можна назвати множинами простої структури [11]. Звичайно «простота» залежить і від наявних обчислювальних ресурсів. Якщо $U-$ множина простої структури, то розглянуті нижче алгоритми безпосередньо можна застосувати для розв'язання задачі керування. Інакше, вони лише моделі деяких чисельних методів, про поведінку яких ми маємо тільки приблизну якісну картину.

\section{3. ВАРІАНТ МЕТОДУ ЛІНЕАРИЗАЦІЇ}

Нехай $f: X \rightarrow \mathbb{R}$ i $\varepsilon>0$. Записом $f(x) \rightarrow \varepsilon-\inf _{x \in X}$ будемо позначати задачу пошуку точок $x^{\prime} \in X$ таких, що $f\left(x^{\prime}\right) \leq \inf _{x \in X} f(x)+\varepsilon$.

Розглянемо задачу оптимального керування (6), (7) та наведений нижче ітераційний процес її розв'язання.

\section{Алгоритм 1. Метод лінеаризації.}

1. Обираємо початкове наближення $u_{0} \in U$. Покладаємо $n=0$.

2. Знаходимо $y_{n} \in H-$ узагальнений розв'язок рівняння: $\mathcal{L} y_{n}=$ $F\left(u_{n}\right)$.

3. Знаходимо спряжений стан $p_{n} \in W_{+}-$розв'язок рівняння

$$
\mathcal{L}^{+} p_{n}=\Phi_{1}^{\prime}\left(y_{n}, u_{n}\right) .
$$


4. Знаходимо $\bar{u}_{n} \in U-$ розв'язок екстремальної задачі

$$
\left(\left(F^{\prime}\left(u_{n}\right)\right)^{*} p_{n}+\Phi_{2}^{\prime}\left(y_{n}, u_{n}\right), u-u_{n}\right)_{V} \rightarrow \varepsilon_{n}-\inf _{u \in U} .
$$

5. Покладаємо $u_{n+1}=u_{n}+\rho_{n}\left(\bar{u}_{n}-u_{n}\right)$, де $\rho_{n} \in(0,1]-$ кроковий множник, $n:=n+1$ і переходимо на крок 2 .

Теорема 4. Нехай

$$
\rho_{n} \in(0,1], \rho_{n} \rightarrow 0, \sum_{n=0}^{\infty} \rho_{n}=+\infty, \varepsilon_{n} \geq 0, \varepsilon_{n} \rightarrow 0 .
$$

Якщо функиіонал $J$ приймае на множсині

$$
U^{*}=\left\{u^{*} \in U:\left(J^{\prime}\left(u^{*}\right), u-u^{*}\right)_{V} \geq 0 \forall u \in U\right\}
$$

не більш нію зліченну кількість значень, то всі граничні точки (які обов'язково існують) послідовності $\left(u_{n}\right)$ утворюють компактну зв'язну підмножину в $U^{*}$, а числова послідовність $\left(J\left(u_{n}\right)\right)$ мае границюо.

Зауваження 6. Нехай $\varepsilon_{n} \searrow 0, \delta_{n}^{\prime} \searrow 0$ і $\delta_{n}^{\prime \prime} \searrow 0$. Аналогічне теоремі 4 твердження про збіжність справджується для наступної моделі алгоритму.

1. Обираємо початкове наближення $u_{0} \in U$. Покладаємо $n=0$.

2. Знаходимо $\tilde{y}_{n} \in H:\left\|\tilde{y}_{n}-y_{n}\right\|_{H} \leq \delta_{n}^{\prime}$, де $y_{n} \in H-$ узагальнений розв'язок рівняння $\mathcal{L} y_{n}=F\left(u_{n}\right)$.

3. Знаходимо $\tilde{p}_{n} \in W_{+}:\left\|\tilde{p}_{n}-p_{n}^{\prime}\right\|_{W_{+}} \leq \delta_{n}^{\prime \prime}$, де $p_{n}^{\prime} \in W_{+}-$розв'язок рівняння

$$
\mathcal{L}^{+} p_{n}^{\prime}=\Phi_{1}^{\prime}\left(\tilde{y}_{n}, u_{n}\right) .
$$

4. Знаходимо $\bar{u}_{n} \in U-$ розв'язок екстремальної задачі

$$
\left(\left(F^{\prime}\left(u_{n}\right)\right)^{*} \tilde{p}_{n}+\Phi_{2}^{\prime}\left(\tilde{y}_{n}, u_{n}\right), u-u_{n}\right)_{V} \rightarrow \varepsilon_{n}-\inf _{u \in U} .
$$

5. Покладаємо $u_{n+1}=u_{n}+\rho_{n}\left(\bar{u}_{n}-u_{n}\right)$, де $\rho_{n} \in(0,1], n:=n+1 \mathrm{i}$ переходимо на крок 2.

Доведення теореми 4. Перевіримо виконання умов теореми про достатні умови збіжності ітераційних алгоритмів оптимізації. Покладемо $W=J$. Функціонал $W$ неперервний на $U$, множина $W^{*}=\left\{W(u): u \in U^{*}\right\}$ має скрізь щільне доповнення. Крім того, функціонал $J$ диференційовний за Фреше, та похідна $J^{\prime}$ задовольняє на $U$ умову Гельдера з показником $\gamma \in$ $(0,1]$. За побудовою всі члени послідовності $\left(u_{n}\right)$ належать компакту $U$.

Розглянемо підпослідовність $\left(u_{n_{k}}\right)$ таку, що $u_{n_{k}} \rightarrow u^{*} \in U^{*}$ при $k \rightarrow \infty$. Маємо

$$
\left\|u_{n_{k}+1}-u_{n_{k}}\right\|_{V}=\rho_{n_{k}}\left\|\bar{u}_{n_{k}}-u_{n_{k}}\right\|_{V} \leq \rho_{n_{k}} \operatorname{diam}(U) \rightarrow 0 \text { при } k \rightarrow \infty .
$$

Нехай $\left(u_{n_{k}}\right)$ - підпослідовність, що збігається до керування $u^{\prime} \notin U^{*}$. Покажемо, що існуе $\delta_{0}>0$ таке, що для всіх $k$ та $\delta \in\left(0, \delta_{0}\right]$ :

$$
\tau_{k}=\min _{n>n_{k}}\left\{n:\left\|u_{n}-u_{n_{k}}\right\|_{V}>\delta\right\}<+\infty .
$$


Припустимо протилежне. Нехай для всіх $\delta_{0}>0$ існує таке $k_{0}=k_{0}\left(\delta_{0}\right) \in$ $\underset{\text { маємо }}{\mathbb{N} \text {, що }}\left\|u_{n}-u_{n_{k_{0}}}\right\|_{V} \leq \delta_{0}$ для всіх $n>n_{k_{0}}$. Тоді з нерівності трикутника

$$
\begin{aligned}
u_{n} \in B_{\delta_{0}}\left(u_{n_{k_{0}}}\right) \Rightarrow u_{n_{k}} \in B_{\delta_{0}}\left(u_{n_{k_{0}}}\right) & \text { для } k>k_{0} \Rightarrow \\
& \Rightarrow u^{\prime} \in B_{\delta_{0}}\left(u_{n_{k_{0}}}\right) \Rightarrow u_{n} \in B_{2 \delta_{0}}\left(u^{\prime}\right)
\end{aligned}
$$

для всіх $n>n_{k_{0}}$.

Оскільки $u^{\prime} \notin U^{*}$, то існують $\lambda>0$ і $\bar{u}^{\prime} \in U$ такі, що

$$
\left(J^{\prime}\left(u^{\prime}\right), \bar{u}^{\prime}-u^{\prime}\right)_{V} \leq-\lambda .
$$

Розглянемо приріст

$$
\begin{gathered}
W\left(u_{n}\right)-W\left(u_{n_{k}}\right)=J\left(u_{n}\right)-J\left(u^{\prime}\right)-J\left(u_{n_{k}}\right)+J\left(u^{\prime}\right)= \\
=\left(J^{\prime}\left(u^{\prime}+\theta^{\prime}\left(u_{n}-u^{\prime}\right)\right), u_{n}-u^{\prime}\right)_{V}- \\
-\left(J^{\prime}\left(u^{\prime}+\theta^{\prime \prime}\left(u_{n_{k}}-u^{\prime}\right)\right), u_{n_{k}}-u^{\prime}\right)_{V}= \\
=\left(J^{\prime}\left(u^{\prime}\right), u_{n}-u^{\prime}\right)_{V}+\left(J^{\prime}\left(u^{\prime}+\theta^{\prime}\left(u_{n}-u^{\prime}\right)\right)-J^{\prime}\left(u^{\prime}\right), u_{n}-u^{\prime}\right)_{V}- \\
-\left(J^{\prime}\left(u^{\prime}\right), u_{n_{k}}-u^{\prime}\right)_{V}+\left(J^{\prime}\left(u^{\prime}\right)-J^{\prime}\left(u^{\prime}+\theta^{\prime \prime}\left(u_{n_{k}}-u^{\prime}\right)\right), u_{n_{k}}-u^{\prime}\right)_{V} \leq \\
\leq\left(J^{\prime}\left(u^{\prime}\right), u_{n}-u_{n_{k}}\right)_{V}+2^{2+\gamma} C \delta_{0}^{1+\gamma}= \\
=\sum_{p=n_{k}}^{n-1} \rho_{p}\left(J^{\prime}\left(u^{\prime}\right), \bar{u}_{p}-u_{p}\right)_{V}+2^{2+\gamma} C \delta_{0}^{1+\gamma},
\end{gathered}
$$

де $n>n_{k}, k>k_{0}$ та $\left\{\theta^{\prime}, \theta^{\prime \prime}\right\} \subset[0,1]$.

У нерівності (9) оцінимо зверху величину $\left(J^{\prime}\left(u^{\prime}\right), \bar{u}_{p}-u_{p}\right)_{V}$. Маємо

$$
\begin{aligned}
& \left(J^{\prime}\left(u^{\prime}\right), \bar{u}_{p}-u_{p}\right)_{V}= \\
& \quad=\left(J^{\prime}\left(u^{\prime}\right)-J^{\prime}\left(u_{p}\right), \bar{u}_{p}-u_{p}\right)_{V}+\left(J^{\prime}\left(u_{p}\right), \bar{u}_{p}-u_{p}\right)_{V} \leq \\
& \quad \leq\left(J^{\prime}\left(u^{\prime}\right)-J^{\prime}\left(u_{p}\right), \bar{u}_{p}-u_{p}\right)_{V}+\left(J^{\prime}\left(u_{p}\right), \bar{u}^{\prime}-u_{p}\right)_{V}+\varepsilon_{p} .
\end{aligned}
$$

Але

$$
\begin{aligned}
\left(J^{\prime}\left(u_{p}\right), \bar{u}^{\prime}-u_{p}\right)_{V}=\left(J^{\prime}\left(u_{p}\right)-\right. & \left.J^{\prime}\left(u^{\prime}\right), \bar{u}^{\prime}-u_{p}\right)_{V}+ \\
& +\left(J^{\prime}\left(u^{\prime}\right), \bar{u}^{\prime}-u^{\prime}\right)_{V}+\left(J^{\prime}\left(u^{\prime}\right), u^{\prime}-u_{p}\right)_{V} .
\end{aligned}
$$

Отже,

$$
\begin{aligned}
\left(J^{\prime}\left(u^{\prime}\right), \bar{u}_{p}-u_{p}\right)_{V} \leq & \left(J^{\prime}\left(u_{p}\right)-J^{\prime}\left(u^{\prime}\right), \bar{u}^{\prime}-\bar{u}_{p}\right)_{V}+ \\
& +\left(J^{\prime}\left(u^{\prime}\right), \bar{u}^{\prime}-u^{\prime}\right)_{V}+\left(J^{\prime}\left(u^{\prime}\right), u^{\prime}-u_{p}\right)_{V}+\varepsilon_{p} .
\end{aligned}
$$

Урахувавши (8), отримаємо оцінку

$$
\begin{aligned}
& \left(J^{\prime}\left(u^{\prime}\right), \bar{u}_{p}-u_{p}\right)_{V} \leq \\
& \quad \leq-\lambda+\operatorname{diam}(U)\left\|J^{\prime}\left(u_{p}\right)-J^{\prime}\left(u^{\prime}\right)\right\|_{V}+2\left\|J^{\prime}\left(u^{\prime}\right)\right\|_{V} \delta_{0}+\varepsilon_{p} \leq \\
& \leq-\lambda+\varepsilon_{p}+\operatorname{diam}(U) C 2^{\gamma} \delta_{0}^{\gamma}+2\left\|J^{\prime}\left(u^{\prime}\right)\right\|_{V} \delta_{0} .
\end{aligned}
$$


Обираючи достатньо мале $\delta_{0}>0$ та велике $k_{0}$, отримуємо $\left(p>n_{k_{0}}\right)$

$$
0<\varepsilon_{p}+\operatorname{diam}(U) C 2^{\gamma} \delta_{0}^{\gamma}+2\left\|J^{\prime}\left(u^{\prime}\right)\right\|_{V} \delta_{0}<\frac{\lambda}{2} .
$$

Звідки $\left(J^{\prime}\left(u^{\prime}\right), \bar{u}_{p}-u_{p}\right)_{V} \leq-\frac{\lambda}{2}, p>n_{k_{0}}$.

Отже, остаточно отримуємо

$$
W\left(u_{n}\right)-W\left(u_{n_{k}}\right) \leq-\frac{\lambda}{2} \sum_{p=n_{k}}^{n-1} \rho_{p}+2^{2+\gamma} C \delta_{0}^{1+\gamma}, n>n_{k} \geq n_{k_{0}} .
$$

Здійснивши граничний перехід в нерівності (10) при $n \rightarrow \infty$ та врахувавши $\sum_{p=n_{k}}^{\infty} \rho_{p}=+\infty$, отримаємо протиріччя з обмеженістю знизу на компактній множині $U$ неперервного функціонала $W$.

Отже, існує $\delta_{0}>0$ таке, що для всіх $k$ i $\delta \in\left(0, \delta_{0}\right]$ :

$$
\tau_{k}=\min _{n>n_{k}}\left\{n:\left\|u_{n}-u_{n_{k}}\right\|_{V}>\delta\right\}<+\infty .
$$

Але, обираючи достатньо мале $\delta_{0}>0$ та велике $k_{0}$, можна повторити доведення оцінки (10) для $n_{k} \leq n \leq \tau_{k}$. 3 іншого боку,

$$
\delta_{0}<\left\|u_{\tau_{k}}-u_{n_{k}}\right\|_{V} \leq \sum_{p=n_{k}}^{\tau_{k}-1}\left\|u_{p+1}-u_{p}\right\|_{V} \leq \operatorname{diam}(U) \sum_{p=n_{k}}^{\tau_{k}-1} \rho_{p} .
$$

Тому $\sum_{p=n_{k}}^{\tau_{k}-1} \rho_{p}>\frac{\delta_{0}}{\operatorname{diam}(U)}$. Ураховуючи останню нерівність в (10), отримуєMO

$$
W\left(u_{\tau_{k}}\right)<W\left(u_{n_{k}}\right)-\frac{\lambda \delta_{0}}{2 \operatorname{diam}(U)}+2^{2+\gamma} C \delta_{0}^{1+\gamma} .
$$

Звідки

$$
\varlimsup_{k \rightarrow \infty} W\left(u_{\tau_{k}}\right)<\lim _{k \rightarrow \infty} W\left(u_{n_{k}}\right) .
$$

Умови теореми про збіжність виконуються, а отже, граничні точки послідовності $\left(u_{n}\right)$ утворюють компактну зв'язну підмножину $U^{*}$ і числова послідовність $\left(J\left(u_{n}\right)\right)$ має границю.

Зауваження 7. Доведемо аналогічний теоремі 4 факт для моделі алгоритму із зауваження 6. Покажемо лише, що для підпослідовності $\left(u_{n_{k}}\right)$, що збігається до керування $u^{\prime} \notin U^{*}$, існує $\delta_{0}>0$ таке, що для всіх $k$ та $\delta \in\left(0, \delta_{0}\right]$

$$
\tau_{k}=\min _{n>n_{k}}\left\{n:\left\|u_{n}-u_{n_{k}}\right\|_{V}>\delta\right\}<+\infty .
$$

Міркуємо від супротивного. Нехай для всіх $\delta_{0}>0$ існує $k_{0}=k_{0}\left(\delta_{0}\right)$ таке, що $\left\|u_{n}-u_{n_{k_{0}}}\right\|_{V} \leq \delta_{0}$ для всіх $n>n_{k_{0}}$. Тоді $u_{n} \in B_{2 \delta_{0}}\left(u^{\prime}\right)$ для всіх $n>n_{k_{0}}$.

Оскільки $u^{\prime} \notin U^{*}$, то існує число $\lambda>0$ таке, що

$$
\min _{u \in U}\left(J^{\prime}\left(u^{\prime}\right), u-u^{\prime}\right)_{V} \leq-2 \lambda<0 .
$$


Розглянемо приріст $J\left(u_{n+1}\right)-J\left(u_{n}\right)$ для $m>n_{k}>n_{k_{0}}$

$$
\begin{gathered}
J\left(u_{m}\right)-J\left(u_{n_{k}}\right)=J\left(u_{m}\right)-J\left(u^{\prime}\right)-J\left(u_{n_{k}}\right)+J\left(u^{\prime}\right)= \\
=\left(J^{\prime}\left(u^{\prime}+\theta^{\prime}\left(u_{m}-u^{\prime}\right)\right), u_{m}-u^{\prime}\right)_{V}- \\
-\left(J^{\prime}\left(u^{\prime}+\theta^{\prime \prime}\left(u_{n_{k}}-u^{\prime}\right)\right), u_{n_{k}}-u^{\prime}\right)_{V}= \\
=\left(J^{\prime}\left(u^{\prime}\right), u_{m}-u^{\prime}\right)_{V}+\left(J^{\prime}\left(u^{\prime}+\theta^{\prime}\left(u_{m}-u^{\prime}\right)\right)-J^{\prime}\left(u^{\prime}\right), u_{m}-u^{\prime}\right)_{V}- \\
-\left(J^{\prime}\left(u^{\prime}\right), u_{n_{k}}-u^{\prime}\right)_{V}+\left(J^{\prime}\left(u^{\prime}\right)-J^{\prime}\left(u^{\prime}+\theta^{\prime \prime}\left(u_{n_{k}}-u^{\prime}\right)\right), u_{n_{k}}-u^{\prime}\right)_{V} \leq \\
\leq \sum_{n=n_{k}}^{m-1} \rho_{n}\left(J^{\prime}\left(u^{\prime}\right), \bar{u}_{n}-u_{n}\right)_{V}+C_{0} \delta_{0}^{1+\gamma},
\end{gathered}
$$

де $\left\{\theta^{\prime}, \theta^{\prime \prime}\right\} \subset[0,1]$.

Оцінимо зверху $\left(J^{\prime}\left(u^{\prime}\right), \bar{u}_{n}-u_{n}\right)_{V}$. Позначимо через $\bar{u}^{\prime} \in U$ розв'язок задачі мінімізації $\left(J^{\prime}\left(u^{\prime}\right), u-u^{\prime}\right)_{V} \rightarrow \inf _{u \in U}$. Маємо

$$
\begin{aligned}
& \left(J^{\prime}\left(u^{\prime}\right), \bar{u}_{n}-u_{n}\right)_{V}=\left(J^{\prime}\left(u^{\prime}\right)-\widetilde{J^{\prime}\left(u_{n}\right)}, \bar{u}_{n}-u_{n}\right)_{V}+ \\
& +\left(\widetilde{J^{\prime}\left(u_{n}\right)}, \bar{u}_{n}-u_{n}\right)_{V} \leq\left(J^{\prime}\left(u^{\prime}\right)-\widetilde{J^{\prime}\left(u_{n}\right)}, \bar{u}_{n}-u_{n}\right)_{V}+\left(\widetilde{J^{\prime}\left(u_{n}\right)}, \bar{u}^{\prime}-u_{n}\right)_{V}+\varepsilon_{n} \text {, }
\end{aligned}
$$

де $\widetilde{J^{\prime}\left(u_{n}\right)}=\left(F^{\prime}\left(u_{n}\right)\right)^{*} \tilde{p}_{n}+\Phi_{2}^{\prime}\left(\tilde{y}_{n}, u_{n}\right)$. Але

$$
\begin{aligned}
\left(\widetilde{J^{\prime}\left(u_{n}\right)}, \bar{u}^{\prime}-u_{n}\right)_{V}=\left(\widetilde{J^{\prime}\left(u_{n}\right)}-J^{\prime}\left(u^{\prime}\right), \bar{u}^{\prime}-u_{n}\right)_{V}+ \\
+\left(J^{\prime}\left(u^{\prime}\right), \bar{u}^{\prime}-u^{\prime}\right)_{V}+\left(J^{\prime}\left(u^{\prime}\right), u^{\prime}-u_{n}\right)_{V} \leq \\
-2 \lambda+\left(\widetilde{J^{\prime}\left(u_{n}\right)}-J^{\prime}\left(u^{\prime}\right), \bar{u}^{\prime}-u_{n}\right)_{V}+\left(J^{\prime}\left(u^{\prime}\right), u^{\prime}-u_{n}\right)_{V} .
\end{aligned}
$$

Звідки

$$
\begin{aligned}
& \left(J^{\prime}\left(u^{\prime}\right), \bar{u}_{n}-u_{n}\right)_{V} \leq \\
& \quad \leq-2 \lambda+\left(\widetilde{J^{\prime}\left(u_{n}\right)}-J^{\prime}\left(u^{\prime}\right), \bar{u}^{\prime}-\bar{u}_{n}\right)_{V}+\left(J^{\prime}\left(u^{\prime}\right), u^{\prime}-u_{n}\right)_{V}+\varepsilon_{n} .
\end{aligned}
$$

Нарешті отримаємо нерівність

$$
\begin{aligned}
& \left(J^{\prime}\left(u^{\prime}\right), \bar{u}_{n}-u_{n}\right)_{V} \leq \\
& \quad \leq-2 \lambda+\operatorname{diam}(U)\left\|\widetilde{J^{\prime}\left(u_{n}\right)}-J^{\prime}\left(u^{\prime}\right)\right\|_{V}+2\left\|J^{\prime}\left(u^{\prime}\right)\right\|_{V} \delta_{0}+\varepsilon_{n} .
\end{aligned}
$$

Оцінимо $\left\|\widetilde{J^{\prime}\left(u_{u}\right)}-J^{\prime}\left(u^{\prime}\right)\right\|_{V}$. Маємо

$$
\begin{aligned}
\left\|\widetilde{J^{\prime}\left(u_{n}\right)}-J^{\prime}\left(u^{\prime}\right)\right\|_{V} \leq \| \widetilde{J^{\prime}\left(u_{n}\right)} & -J^{\prime}\left(u_{n}\right)\left\|_{V}+\right\| J^{\prime}\left(u_{n}\right)-J^{\prime}\left(u^{\prime}\right) \|_{V} \leq \\
& \leq\left\|\widetilde{J^{\prime}\left(u_{n}\right)}-J^{\prime}\left(u_{n}\right)\right\|_{V}+C_{1}\left\|u_{n}-u^{\prime}\right\|_{V}^{\gamma} .
\end{aligned}
$$


Далі

$$
\begin{aligned}
& \left\|\widetilde{J^{\prime}\left(u_{n}\right)}-J^{\prime}\left(u_{n}\right)\right\|_{V} \leq \\
& \quad \leq\left\|\left(F^{\prime}\left(u_{n}\right)\right)^{*} \tilde{p}_{n}+\Phi_{2}^{\prime}\left(\tilde{y}_{n}, u_{n}\right)-\left(F^{\prime}\left(u_{n}\right)\right)^{*} p_{n}-\Phi_{2}^{\prime}\left(y_{n}, u_{n}\right)\right\|_{V} \leq \\
& \leq\left\|F^{\prime}\left(u_{n}\right)\right\|\left\|\tilde{p}_{n}-p_{n}\right\|_{W_{+}}+\left\|\Phi_{2}^{\prime}\left(\tilde{y}_{n}, u_{n}\right)-\Phi_{2}^{\prime}\left(y_{n}, u_{n}\right)\right\|_{V} \leq \\
& \quad \leq C_{1}\left\|\tilde{p}_{n}-p_{n}^{\prime}\right\|_{W_{+}}+C_{1}\left\|p_{n}^{\prime}-p_{n}\right\|_{W_{+}}+C_{2}\left\|\tilde{y}_{n}-y_{n}\right\|_{H}^{\gamma} \leq \\
& \leq C_{1} \delta_{n}^{\prime \prime}+C_{2}\left(\delta_{n}^{\prime}\right)^{\gamma}+C_{3}\left\|\Phi_{1}^{\prime}\left(\tilde{y}_{n}, u_{n}\right)-\Phi_{1}^{\prime}\left(y_{n}, u_{n}\right)\right\|_{H^{-}} \leq C_{1} \delta_{n}^{\prime \prime}+C_{4}\left(\delta_{n}^{\prime}\right)^{\gamma},
\end{aligned}
$$

де $p_{n} \in W_{+}$- розв'язок спряженої задачі $\mathcal{L}^{+} p_{n}=\Phi_{1}^{\prime}\left(y_{n}, u_{n}\right)$.

Отже,

$\left(J^{\prime}\left(u^{\prime}\right), \bar{u}_{n}-u_{n}\right)_{V} \leq-2 \lambda+\operatorname{diam}(U)\left(C_{1} \delta_{n}^{\prime \prime}+C_{4}\left(\delta_{n}^{\prime}\right)^{\gamma}\right)+2\left\|J^{\prime}\left(u^{\prime}\right)\right\|_{V} \delta_{0}+\varepsilon_{n}$.

Обираючи достатньо мале $\delta_{0}>0$ та велике $k_{0}$, отримуємо $\left(n>n_{k_{0}}\right)$

$$
0<\operatorname{diam}(U)\left(C_{1} \delta_{n}^{\prime \prime}+C_{4}\left(\delta_{n}^{\prime}\right)^{\gamma}\right)+2\left\|J^{\prime}\left(u^{\prime}\right)\right\|_{V} \delta_{0}+\varepsilon_{n}<\lambda .
$$

Звідки $\left(J^{\prime}\left(u^{\prime}\right), \bar{u}_{n}-u_{n}\right)_{V} \leq-\lambda, n>n_{k_{0}}$.

Остаточно отримуємо

$$
J\left(u_{m}\right)-J\left(u_{n_{k}}\right) \leq-\lambda \sum_{n=n_{k}}^{m-1} \rho_{n}+C_{0} \delta_{0}^{1+\gamma}, m>n_{k} \geq n_{k_{0}} .
$$

Після граничного переходу в нерівності при $m \rightarrow \infty\left(\sum_{n=n_{k}}^{\infty} \rho_{n}=+\infty\right)$ маємо протиріччя з обмеженістю знизу на множині $U$ функціонала $J$.

Розглянемо тепер більш реалістичну ситуацію, коли точність $\varepsilon_{n}$ розв'язання допоміжної задачі мінімізації лінійної форми не прямує до нуля.

Теорема 5. Нехай

$$
\rho_{n} \in(0,1], \rho_{n} \rightarrow 0, \sum_{n=0}^{\infty} \rho_{n}=+\infty, \varepsilon_{n} \in[0, \bar{\varepsilon}], \bar{\varepsilon} \geq 0 .
$$

Якщо функиіонал $J$ приймае на множині

$$
U_{\bar{\varepsilon}}^{*}=\left\{u^{*} \in U:\left(J^{\prime}\left(u^{*}\right), u-u^{*}\right)_{V} \geq-\bar{\varepsilon}, \forall u \in U\right\}
$$

не більш ніж зліченну кількість значень, то всі граничні точки (які обов'язково існують) послідовності $\left(u_{n}\right)$, що генеруетъся алгоритмом 1, утворюють компактну зв'язну підмножсиу в $U_{\bar{\varepsilon}}^{*}$, а числова послідовність $\left(J\left(u_{n}\right)\right)$ мае границ,ю.

Доведення. Перевіримо виконання умов теореми про достатні умови збіжності ітераційних алгоритмів оптимізації. Покладемо $W=J$. Функціонал $W$ неперервний на множині $U$, множина $W^{*}=\left\{W(u): u \in U_{\bar{\varepsilon}}^{*}\right\}$ має скрізь щільне доповнення. Члени послідовності $\left(u_{n}\right)$ лежать в компакті $U$. Розглянемо підпослідовність $\left(u_{n_{k}}\right)$ таку, що $u_{n_{k}} \rightarrow u^{*} \in U_{\bar{\varepsilon}}^{*}$ при $k \rightarrow \infty$. Маємо

$$
\left\|u_{n_{k}+1}-u_{n_{k}}\right\|_{V}=\rho_{n_{k}}\left\|\bar{u}_{n_{k}}-u_{n_{k}}\right\|_{V} \leq \rho_{n_{k}} \operatorname{diam}(U) \rightarrow 0 \text { при } k \rightarrow \infty .
$$


Нехай $\left(u_{n_{k}}\right)$ - підпослідовність, що збігається до керування $u^{\prime} \notin U_{\bar{\varepsilon}}^{*}$. Покажемо, що існує таке $\delta_{0}>0$, що для всіх $k$ та $\delta \in\left(0, \delta_{0}\right]$

$$
\tau_{k}=\min _{n>n_{k}}\left\{n:\left\|u_{n}-u_{n_{k}}\right\|_{V}>\delta\right\}<+\infty .
$$

Від супротивного. Нехай для всіх $\delta_{0}>0$ існує таке $k_{0}=k_{0}\left(\delta_{0}\right)$, що $\left\|u_{n}-u_{n_{k_{0}}}\right\|_{V} \leq \delta_{0}$ для всіх $n>n_{k_{0}}$. Тоді з нерівностей

$$
\left\|u_{n}-u_{n_{k_{0}}}\right\|_{V} \leq \delta_{0},\left\|u_{n_{k}}-u_{n_{k_{0}}}\right\|_{V} \leq \delta_{0} \quad \forall k>k_{0} \Rightarrow\left\|u^{\prime}-u_{n_{k_{0}}}\right\|_{V} \leq \delta_{0}
$$

випливає, що $u_{n} \in B_{2 \delta_{0}}\left(u^{\prime}\right)$ для всіх $n>n_{k_{0}}$.

Оскільки $u^{\prime} \notin U_{\bar{\varepsilon}}^{*}$, то існують $\lambda>0$ і $\bar{u}^{\prime} \in U$ такі, що

$$
\left(J^{\prime}\left(u^{\prime}\right), \bar{u}^{\prime}-u^{\prime}\right)_{V} \leq-\lambda-\bar{\varepsilon} .
$$

Розглянемо приріст

$$
\begin{gathered}
W\left(u_{n}\right)-W\left(u_{n_{k}}\right)=J\left(u_{n}\right)-J\left(u^{\prime}\right)-J\left(u_{n_{k}}\right)+J\left(u^{\prime}\right)= \\
=\left(J^{\prime}\left(u^{\prime}+\theta^{\prime}\left(u_{n}-u^{\prime}\right)\right), u_{n}-u^{\prime}\right)_{V}- \\
-\left(J^{\prime}\left(u^{\prime}+\theta^{\prime \prime}\left(u_{n_{k}}-u^{\prime}\right)\right), u_{n_{k}}-u^{\prime}\right)_{V}= \\
=\left(J^{\prime}\left(u^{\prime}\right), u_{n}-u^{\prime}\right)_{V}+\left(J^{\prime}\left(u^{\prime}+\theta^{\prime}\left(u_{n}-u^{\prime}\right)\right)-J^{\prime}\left(u^{\prime}\right), u_{n}-u^{\prime}\right)_{V}- \\
-\left(J^{\prime}\left(u^{\prime}\right), u_{n_{k}}-u^{\prime}\right)_{V}+\left(J^{\prime}\left(u^{\prime}\right)-J^{\prime}\left(u^{\prime}+\theta^{\prime \prime}\left(u_{n_{k}}-u^{\prime}\right)\right), u_{n_{k}}-u^{\prime}\right)_{V} \leq \\
\leq\left(J^{\prime}\left(u^{\prime}\right), u_{n}-u_{n_{k}}\right)_{V}+2^{2+\gamma} C \delta_{0}^{1+\gamma}= \\
=\sum_{p=n_{k}}^{n-1} \rho_{p}\left(J^{\prime}\left(u^{\prime}\right), \bar{u}_{p}-u_{p}\right)_{V}+2^{2+\gamma} C \delta_{0}^{1+\gamma}, \quad
\end{gathered}
$$

де $n>n_{k}, k>k_{0},\left\{\theta^{\prime}, \theta^{\prime \prime}\right\} \subset[0,1]$.

$\mathrm{У}$ нерівності (12) оцінимо зверху величину $\left(J^{\prime}\left(u^{\prime}\right), \bar{u}_{p}-u_{p}\right)_{V}$. Маємо

$$
\begin{aligned}
& \left(J^{\prime}\left(u^{\prime}\right), \bar{u}_{p}-u_{p}\right)_{V}= \\
& \quad=\left(J^{\prime}\left(u^{\prime}\right)-J^{\prime}\left(u_{p}\right), \bar{u}_{p}-u_{p}\right)_{V}+\left(J^{\prime}\left(u_{p}\right), \bar{u}_{p}-u_{p}\right)_{V} \leq \\
& \quad \leq\left(J^{\prime}\left(u^{\prime}\right)-J^{\prime}\left(u_{p}\right), \bar{u}_{p}-u_{p}\right)_{V}+\left(J^{\prime}\left(u_{p}\right), \bar{u}^{\prime}-u_{p}\right)_{V}+\varepsilon_{p} .
\end{aligned}
$$

Але

$$
\begin{gathered}
\left(J^{\prime}\left(u_{p}\right), \bar{u}^{\prime}-u_{p}\right)_{V}=\left(J^{\prime}\left(u_{p}\right)-J^{\prime}\left(u^{\prime}\right), \bar{u}^{\prime}-u_{p}\right)_{V}+ \\
+\left(J^{\prime}\left(u^{\prime}\right), \bar{u}^{\prime}-u^{\prime}\right)_{V}+\left(J^{\prime}\left(u^{\prime}\right), u^{\prime}-u_{p}\right)_{V} \cdot
\end{gathered}
$$

Отже,

$$
\begin{aligned}
\left(J^{\prime}\left(u^{\prime}\right), \bar{u}_{p}-u_{p}\right)_{V} \leq & \left(J^{\prime}\left(u_{p}\right)-J^{\prime}\left(u^{\prime}\right), \bar{u}^{\prime}-\bar{u}_{p}\right)_{V}+ \\
& +\left(J^{\prime}\left(u^{\prime}\right), \bar{u}^{\prime}-u^{\prime}\right)_{V}+\left(J^{\prime}\left(u^{\prime}\right), u^{\prime}-u_{p}\right)_{V}+\varepsilon_{p} .
\end{aligned}
$$


Урахувавши (11), отримаємо оцінку

$$
\begin{aligned}
& \left(J^{\prime}\left(u^{\prime}\right), \bar{u}_{p}-u_{p}\right)_{V} \leq \\
& \quad \leq-\lambda-\bar{\varepsilon}+\operatorname{diam}(U)\left\|J^{\prime}\left(u_{p}\right)-J^{\prime}\left(u^{\prime}\right)\right\|_{V}+2\left\|J^{\prime}\left(u^{\prime}\right)\right\|_{V} \delta_{0}+\varepsilon_{p} \leq \\
& \quad \leq-\lambda-\bar{\varepsilon}+\varepsilon_{p}+\operatorname{diam}(U) C 2^{\gamma} \delta_{0}^{\gamma}+2\left\|J^{\prime}\left(u^{\prime}\right)\right\|_{V} \delta_{0} .
\end{aligned}
$$

Обираючи достатньо мале $\delta_{0}>0$ та велике $k_{0}$, отримуємо

$$
\begin{aligned}
-\bar{\varepsilon}+\varepsilon_{p}+\operatorname{diam}(U) C 2^{\gamma} \delta_{0}^{\gamma}+2 \| & J^{\prime}\left(u^{\prime}\right) \|_{V} \delta_{0} \leq \\
& \leq \operatorname{diam}(U) C 2^{\gamma} \delta_{0}^{\gamma}+2\left\|J^{\prime}\left(u^{\prime}\right)\right\|_{V} \delta_{0}<\frac{\lambda}{2} .
\end{aligned}
$$

Звідки $\left(J^{\prime}\left(u^{\prime}\right), \bar{u}_{p}-u_{p}\right)_{V} \leq-\frac{\lambda}{2}, p>n_{k_{0}}$.

Остаточно маємо

$$
W\left(u_{n}\right)-W\left(u_{n_{k}}\right) \leq-\frac{\lambda}{2} \sum_{p=n_{k}}^{n-1} \rho_{p}+2^{2+\gamma} C \delta_{0}^{1+\gamma}, \quad n>n_{k} \geq n_{k_{0}} .
$$

Здійснивши граничний перехід в (13) при $n \rightarrow \infty$ та врахувавши $\sum_{p=n_{k}}^{\infty} \rho_{p}=$ $+\infty$, отримаємо протиріччя з обмеженістю на компактній множині $U$ неперервного функціонала $W$.

Отже, існуе $\delta_{0}>0$ таке, що для всіх $k$ i $\delta \in\left(0, \delta_{0}\right]$ :

$$
\tau_{k}=\min _{n>n_{k}}\left\{n:\left\|u_{n}-u_{n_{k}}\right\|_{V}>\delta\right\}<+\infty .
$$

Але, обираючи достатньо мале $\delta_{0}>0$ та велике $k_{0}$, можна повторити доведення нерівності (13) для $n_{k} \leq n \leq \tau_{k}$. 3 іншого боку,

$$
\delta_{0}<\left\|u_{\tau_{k}}-u_{n_{k}}\right\|_{V} \leq \sum_{p=n_{k}}^{\tau_{k}-1}\left\|u_{p+1}-u_{p}\right\|_{V} \leq \operatorname{diam}(U) \sum_{p=n_{k}}^{\tau_{k}-1} \rho_{p} .
$$

Тому $\sum_{p=n_{k}}^{\tau_{k}-1} \rho_{p}>\frac{\delta_{0}}{\operatorname{diam}(U)}$. Підставивши останню оцінку в (13), одержуємо

$$
W\left(u_{\tau_{k}}\right)-W\left(u_{n_{k}}\right)<-\frac{\lambda \delta_{0}}{2 \operatorname{diam}(U)}+2^{2+\gamma} C \delta_{0}^{1+\gamma} .
$$

Звідки

$$
\limsup _{k \rightarrow \infty} W\left(u_{\tau_{k}}\right)<\lim _{k \rightarrow \infty} W\left(u_{n_{k}}\right) .
$$

Таким чином, умови абстрактної теореми про збіжність виконуються.

Зауваження 8. В алгоритмі 1 на 3-му кроці можна розглянути таку допоміжну задачу мінімізації

$$
\left(Q_{n}, u-u_{n}\right)_{V} \rightarrow \varepsilon_{n}-\inf _{u \in U},
$$

де $Q_{n}=\left(1-\alpha_{n}\right) Q_{n-1}+\alpha_{n}\left\{\left(F^{\prime}\left(u_{n}\right)\right)^{*} p_{n}+\Phi_{2}^{\prime}\left(y_{n}, u_{n}\right)\right\}$. Якщо коефіцієнти усереднення $\alpha_{n}$ обирати з дотриманням умов $\alpha_{n} \in(0,1), \sum_{n=1}^{\infty} \alpha_{n}=+\infty$, $\frac{\rho_{n}^{\gamma}}{\alpha_{n}} \rightarrow 0$ при $n \rightarrow \infty$, то для цієї модифікації будуть справедливі аналоги теорем 4 і 5. 


\section{4. ВАРІАНТ МЕТОДУ ПРОЕКЦІЇ ГРАДІЕНТА}

Розглянемо ітераційний процес розв'язання задачі оптимального керування (6), (7) з наближеною мінімізацією деякого квадратичного функціоналу на кожному кроці.

\section{Алгоритм 2. Метод проекції градієнта.}

1. Обираємо початкове наближення $u_{0} \in U$. Покладаємо $n=0$.

2. Знаходимо $y_{n} \in H-$ узагальнений розв'язок рівняння $\mathcal{L} y_{n}=F\left(u_{n}\right)$.

3. Знаходимо спряжений стан $p_{n} \in W_{+}$- розв'язок рівняння

$$
\mathcal{L}^{+} p_{n}=\Phi_{1}^{\prime}\left(y_{n}, u_{n}\right) .
$$

4. Знаходимо $\bar{u}_{n} \in U-$ розв'язок екстремальної задачі

$$
\left(\left(F^{\prime}\left(u_{n}\right)\right)^{*} p_{n}+\Phi_{2}^{\prime}\left(y_{n}, u_{n}\right), u-u_{n}\right)_{V}+\frac{1}{2 \alpha_{n}}\left\|u-u_{n}\right\|_{V}^{2} \rightarrow \varepsilon_{n}-\inf _{u \in U} .
$$

5. Покладаємо $u_{n+1}=u_{n}+\rho_{n}\left(\bar{u}_{n}-u_{n}\right)$, де $\rho_{n} \in(0,1]-$ кроковий множник, $n:=n+1$ і переходимо на крок 2 .

Зауваження 9. Алгоритм 2 - один з варіантів відомого методу проекції градієнта. Дійсно, при $\varepsilon_{n}=0$ задача кроку 4 рівносильна задачі мінімізації на множині $U$ функціонала

$$
\begin{aligned}
& \frac{\alpha_{n}^{2}}{2}\left\|\left(F^{\prime}\left(u_{n}\right)\right)^{*} p_{n}+\Phi_{2}^{\prime}\left(y_{n}, u_{n}\right)\right\|_{V}^{2}+ \\
& \quad+\alpha_{n}\left(\left(F^{\prime}\left(u_{n}\right)\right)^{*} p_{n}+\Phi_{2}^{\prime}\left(y_{n}, u_{n}\right), u-u_{n}\right)_{V}+\frac{1}{2}\left\|u-u_{n}\right\|_{V}^{2}= \\
& \quad=\frac{1}{2}\left\|u-\left(u_{n}-\alpha_{n}\left(F^{\prime}\left(u_{n}\right)\right)^{*} p_{n}+\alpha_{n} \Phi_{2}^{\prime}\left(y_{n}, u_{n}\right)\right)\right\|_{V}^{2},
\end{aligned}
$$

тобто, знаходженню ортогональної проекції вектора $u_{n}-\alpha_{n} J^{\prime}\left(u_{n}\right)$ на множину $U$.

Теорема 6. Нехай $\rho_{n} \in[\rho, \bar{\rho}] \subseteq(0,1], \alpha_{n} \rightarrow 0, \sum_{n=0}^{\infty} \alpha_{n}=+\infty, \varepsilon_{n}>0$, $\frac{\varepsilon_{n}}{\alpha_{n}} \rightarrow 0$. Якщо функціонал $J$ приймае на множсиі

$$
U^{*}=\left\{u^{*} \in U:\left(J^{\prime}\left(u^{*}\right), u-u^{*}\right)_{V} \geq 0, \forall u \in U\right\}
$$

не більш нію зліченну кількість значень, то всі граничні точки (лкі обов'язково існують) послідовності $\left(u_{n}\right)$ утворюють компактну зв'язну підмножину в $U^{*}$, а числова послідовність $\left(J\left(u_{n}\right)\right)$ мае границю.

Доведення. Перевіримо виконання умов теореми про достатні умови збіжності ітераційних алгоритмів оптимізації. За побудовою усі члени послідовності $\left(u_{n}\right)$ належать компакту $U$. Покладемо $W=J$.

Розглянемо підпослідовність $\left(u_{n_{k}}\right)$ таку, що $u_{n_{k}} \rightarrow u^{*} \in U^{*}$ при $k \rightarrow \infty$. Оцінимо $\left\|u_{n_{k}+1}-u_{n_{k}}\right\|_{V}$. Функціонал

$$
u \mapsto R_{n_{k}}(u)=\left(\left(F^{\prime}\left(u_{n_{k}}\right)\right)^{*} p_{n_{k}}+\Phi_{2}^{\prime}\left(y_{n_{k}}, u_{n_{k}}\right), u-u_{n_{k}}\right)_{V}+\frac{1}{2 \alpha_{n_{k}}}\left\|u-u_{n_{k}}\right\|_{V}^{2}
$$

сильно опуклий $\left(\frac{1}{2 \alpha_{n_{k}}}>0-\right.$ стала сильної опуклості). Покладемо

$$
\tilde{u}_{n_{k}}=\arg \min _{u \in U} R_{n_{k}}(u)=\Pi_{U}\left(u_{n_{k}}-\alpha_{n_{k}} J^{\prime}\left(u_{n_{k}}\right)\right),
$$


де $\Pi_{U}(\cdot)$ - оператор проектування на множину $U$. Маємо

$$
\frac{1}{2 \alpha_{n_{k}}}\left\|\bar{u}_{n_{k}}-\tilde{u}_{n_{k}}\right\|_{V}^{2} \leq R\left(\bar{u}_{n_{k}}\right)-R\left(\tilde{u}_{n_{k}}\right) \leq \varepsilon_{n_{k}} .
$$

Отже,

$$
\begin{gathered}
\left\|u_{n_{k}+1}-u_{n_{k}}\right\|_{V}=\rho_{n_{k}}\left\|\bar{u}_{n_{k}}-u_{n_{k}}\right\|_{V} \leq \bar{\rho}\left\|\bar{u}_{n_{k}}-u_{n_{k}}\right\|_{V} \leq \\
\leq\left\|\bar{u}_{n_{k}}-\tilde{u}_{n_{k}}\right\|_{V}+\left\|\tilde{u}_{n_{k}}-u_{n_{k}}\right\|_{V} \leq \\
\leq \sqrt{2 \alpha_{n_{k}} \varepsilon_{n_{k}}}+\left\|u_{n_{k}}-\alpha_{n_{k}} J^{\prime}\left(u_{n_{k}}\right)-u_{n_{k}}\right\|_{V} \leq \\
\leq \sqrt{2 \alpha_{n_{k}} \varepsilon_{n_{k}}}+C_{0} \alpha_{n_{k}} \rightarrow 0 \text { при } k \rightarrow \infty,
\end{gathered}
$$

де $C_{0}=\sup _{u \in U}\left\|J^{\prime}(u)\right\|_{V}<+\infty$.

Нехай $\left(u_{n_{k}}\right)$ - підпослідовність, що збігається до керування $u^{\prime} \notin U^{*}$. Покажемо, що існує таке $\delta_{0}>0$, що для всіх $k$ i $\delta \in\left(0, \delta_{0}\right]$ :

$$
\tau_{k}=\min _{n>n_{k}}\left\{n:\left\|u_{n}-u_{n_{k}}\right\|_{V}>\delta\right\}<+\infty .
$$

Від супротивного. Нехай для всіх $\delta_{0}>0$ існує $k_{0}=k_{0}\left(\delta_{0}\right)$ таке, що $\| u_{n}-$ $u_{n_{k_{0}}} \|_{V} \leq \delta_{0}$ для всіх $n>n_{k_{0}}$. Тоді з нерівностей

$$
\left\|u_{n}-u_{n_{k_{0}}}\right\|_{V} \leq \delta_{0},\left\|u_{n_{k}}-u_{n_{k_{0}}}\right\|_{V} \leq \delta_{0} \quad \forall k>k_{0} \Rightarrow\left\|u^{\prime}-u_{n_{k_{0}}}\right\|_{V} \leq \delta_{0}
$$

випливає, що $u_{n} \in B_{2 \delta_{0}}\left(u^{\prime}\right)$ для всіх $n>n_{k_{0}}$.

Використовуючи нерівність

$$
J(v)-J(u)-\left(J^{\prime}(u), v-u\right)_{V} \leq \frac{C_{1}}{\gamma+1}\|v-u\|_{V}^{\gamma+1},
$$

де $C_{1} \geq 0$ - константа з умови Гельдера для похідної $J^{\prime}(\cdot)$, оцінимо приріст $W\left(u_{n+1}\right)-W\left(u_{n}\right)$. Маємо

$$
\begin{gathered}
W\left(u_{n+1}\right)-W\left(u_{n}\right) \leq\left(J^{\prime}\left(u_{n}\right), u_{n+1}-u_{n}\right)_{V}+\frac{C_{1}}{\gamma+1}\left\|u_{n+1}-u_{n}\right\|_{V}^{\gamma+1}= \\
=\rho_{n}\left(J^{\prime}\left(u_{n}\right), \bar{u}_{n}-u_{n}\right)_{V}+\frac{C_{1}}{\gamma+1} \rho_{n}^{\gamma+1}\left\|\bar{u}_{n}-u_{n}\right\|_{V}^{\gamma+1}= \\
=\rho_{n}\left\{\left(J^{\prime}\left(u_{n}\right), \bar{u}_{n}-u_{n}\right)_{V}+\frac{1}{2 \alpha_{n}}\left\|\bar{u}_{n}-u_{n}\right\|_{V}^{2}\right\}+ \\
+\frac{C_{1}}{\gamma+1} \rho_{n}^{\gamma+1}\left\|\bar{u}_{n}-u_{n}\right\|_{V}^{\gamma+1}-\frac{\rho_{n}}{2 \alpha_{n}}\left\|\bar{u}_{n}-u_{n}\right\|_{V}^{2} \leq \\
\leq \rho_{n}\left\{\varepsilon_{n}+\min _{u \in U} R_{n}(u)\right\}+\frac{C_{1}}{\gamma+1} \rho_{n}^{\gamma+1}\left\|\bar{u}_{n}-u_{n}\right\|_{V}^{\gamma+1} .
\end{gathered}
$$

Оскільки $u^{\prime} \notin U^{*}$, то існує таке $\lambda>0$, що

$$
\min _{u \in U}\left(J^{\prime}\left(u^{\prime}\right), u-u^{\prime}\right)_{V} \leq-2 \lambda<0 .
$$

Далі, існує $\delta>0$ таке, що для довільної точки $u^{\prime \prime} \in O_{\delta}\left(u^{\prime}\right)$ виконується нерівність

$$
\min _{u \in U}\left(J^{\prime}\left(u^{\prime \prime}\right), u-u^{\prime \prime}\right)_{V} \leq-\lambda<0 .
$$


Візьмемо $2 \delta_{0}<\delta$, тоді $u_{n} \in B_{2 \delta_{0}}\left(u^{\prime}\right) \subseteq O_{\delta}\left(u^{\prime}\right)$. Позначимо через $\overline{\bar{u}}_{n} \in U$ розв'язок задачі мінімізації

$$
\left(J^{\prime}\left(u_{n}\right), u-u_{n}\right)_{V} \rightarrow \inf _{u \in U} .
$$

Для $\overline{\bar{u}}_{n} \in U$ виконується нерівність

$$
-\left\|J^{\prime}\left(u_{n}\right)\right\|_{V}\left\|\overline{\bar{u}}_{n}-u_{n}\right\|_{V} \leq\left(J^{\prime}\left(u_{n}\right), \overline{\bar{u}}_{n}-u_{n}\right)_{V} \leq-\lambda<0
$$

тобто,

$$
\frac{1}{\left\|\overline{\bar{u}}_{n}-u_{n}\right\|_{V}} \leq \frac{\left\|J^{\prime}\left(u_{n}\right)\right\|_{V}}{\lambda} \leq \frac{C_{0}}{\lambda} .
$$

Обираючи достатньо велике $k_{0}$, ми можемо досягти для всіх $n>n_{k_{0}}$ виконання нерівності $\alpha_{n} \leq \frac{\lambda}{C_{0}^{2}}$. Розглянемо точку $u_{n}^{\prime \prime}=u_{n}+\mu_{n}\left(\overline{\bar{u}}_{n}-u_{n}\right)$, де $\mu_{n}=\frac{\lambda \alpha_{n}}{\left\|\overline{\bar{u}}_{n}-u_{n}\right\|_{V}^{2}} \in(0,1]$. Очевидно, що $u_{n}^{\prime \prime} \in U$. Підставимо точку $u_{n}^{\prime \prime} \in U$ у праву частину оцінки (14). Отримаємо ланцюжок нерівностей

$$
\begin{gathered}
W\left(u_{n+1}\right)-W\left(u_{n}\right) \leq \\
\leq \rho_{n}\left\{\varepsilon_{n}+\left(J^{\prime}\left(u_{n}\right), u_{n}^{\prime \prime}-u_{n}\right)_{V}+\frac{1}{2 \alpha_{n}}\left\|u_{n}^{\prime \prime}-u_{n}\right\|_{V}^{2}\right\}+ \\
\quad+\frac{C_{1}}{\gamma+1} \rho_{n}^{\gamma+1}\left\|\bar{u}_{n}-u_{n}\right\|_{V}^{\gamma+1}= \\
=\rho_{n}\left\{\varepsilon_{n}+\mu_{n}\left(J^{\prime}\left(u_{n}\right), \overline{\bar{u}}_{n}-u_{n}\right)_{V}+\frac{\mu_{n}^{2}}{2 \alpha_{n}}\left\|\overline{\bar{u}}_{n}-u_{n}\right\|_{V}^{2}\right\}+ \\
\quad+\frac{C_{1}}{\gamma+1} \rho_{n}^{\gamma+1}\left\|\bar{u}_{n}-u_{n}\right\|_{V}^{\gamma+1} \leq \\
\leq \rho_{n}\left\{\varepsilon_{n}-\mu_{n} \lambda+\frac{\mu_{n}^{2}}{2 \alpha_{n}}\left\|\overline{\bar{u}}_{n}-u_{n}\right\|_{V}^{2}\right\}+\frac{C_{1}}{\gamma+1}\left(\alpha_{n} \sqrt{\frac{2 \varepsilon_{n}}{\alpha_{n}}}+C_{0} \alpha_{n}\right)^{\gamma+1}= \\
=\rho_{n}\left\{\varepsilon_{n}-\frac{\lambda^{2} \alpha_{n}}{2\left\|\overline{\bar{u}}_{n}-u_{n}\right\|_{V}^{2}}\right\}+\frac{C_{1}}{\gamma+1}\left(\alpha_{n} \sqrt{\frac{2 \varepsilon_{n}}{\alpha_{n}}}+C_{0} \alpha_{n}\right)^{\gamma+1} \leq \\
\leq-\frac{\rho}{2}\left(\frac{\lambda}{\operatorname{diam}(U)}\right)^{2} \alpha_{n}+\varepsilon_{n}+\frac{C_{1}}{\gamma+1} \alpha_{n}^{\gamma+1}\left(\sqrt{\frac{2 \varepsilon_{n}}{\alpha_{n}}}+C_{0}\right)^{\gamma+1} .
\end{gathered}
$$

Оскільки $\alpha_{n} \rightarrow 0, \varepsilon_{n} \rightarrow 0$ і $\varepsilon_{n} \alpha_{n}^{-1} \rightarrow 0$, то, обираючи достатньо мале $\delta_{0}>0$ та велике $k_{0}$, отримуємо

$$
W\left(u_{n+1}\right)-W\left(u_{n}\right) \leq-\frac{\rho}{4}\left(\frac{\lambda}{\operatorname{diam}(U)}\right)^{2} \alpha_{n}, \quad n>n_{k_{0}} .
$$

Остаточно маємо

$$
W\left(u_{n}\right)-W\left(u_{n_{k}}\right) \leq-\frac{\rho}{4}\left(\frac{\lambda}{\operatorname{diam}(U)}\right)^{2} \sum_{p=n_{k}}^{n-1} \alpha_{p}, n>n_{k}>n_{k_{0}} .
$$

Здійснивши граничний перехід в нерівності (15) при $n \rightarrow \infty$ і врахувавши $\sum_{p=n_{k}}^{\infty} \alpha_{p}=+\infty$, отримаємо протиріччя з обмеженістю на компакті $U$ функціонала $W$. 
Отже, існує $\delta_{0}>0$ таке, що для всіх $k$ i $\delta \in\left(0, \delta_{0}\right]$ :

$$
\tau_{k}=\min _{n>n_{k}}\left\{n:\left\|u_{n}-u_{n_{k}}\right\|_{V}>\delta\right\}<+\infty .
$$

Але обираючи достатньо мале $\delta_{0}>0$ та велике $k_{0}$, можна повторити доведення оцінки (15) для $n_{k} \leq n \leq \tau_{k}$. 3 іншого боку

$\delta_{0}<\left\|u_{\tau_{k}}-u_{n_{k}}\right\|_{V} \leq \sum_{p=n_{k}}^{\tau_{k}-1}\left\|u_{p+1}-u_{p}\right\|_{V} \leq \sum_{p=n_{k}}^{\tau_{k}-1}\left(\sqrt{2 \alpha_{p} \varepsilon_{p}}+C_{0} \alpha_{p}\right) \leq C_{2} \sum_{p=n_{k}}^{\tau_{k}-1} \alpha_{p}$.

Тому $\sum_{p=n_{k}}^{\tau_{k}-1} \alpha_{p}>\frac{\delta_{0}}{C_{2}}>0$. Враховуючи останню нерівність в (15), отримуєMO

$$
W\left(u_{\tau_{k}}\right)-W\left(u_{n_{k}}\right)<-\frac{\rho}{4 C_{2}}\left(\frac{\lambda}{\operatorname{diam}(U)}\right)^{2} \delta_{0} .
$$

Звідки $\lim \sup _{k \rightarrow \infty} W\left(u_{\tau_{k}}\right)<\lim _{k \rightarrow \infty} W\left(u_{n_{k}}\right)$. Отже, умови теореми про збіжність виконуються. Тому граничні точки послідовності $\left(u_{n}\right)$ утворюють компактну зв'язну підмножину $U^{*}$ і числова послідовність $\left(J\left(u_{n}\right)\right)$ має границю.

\section{5. МЕТОД ЛІНЕАРИЗАЦІЇ З ДОВІРЧОЮ ОБЛАСТЮ}

Розглянемо задачу оптимального керування

$$
\begin{gathered}
J(u)=\Phi(y(u), u) \rightarrow \inf _{u \in U}, \\
\mathcal{L} y(u)=F(u), u \in U,
\end{gathered}
$$

де $F: V \rightarrow W_{+}^{-}, U-$ компактна та опукла множина допустимих керувань із гільбертового простору керувань $V$. Це типова ситуація в імпульсноточковому керуванні розподіленими системами $[3,5]$.

Нехай є дві числові послідовності $\left(\alpha_{n}\right)$ і $\left(\beta_{n}\right)$ такі, що $0<\alpha_{n} \leq \beta_{n}$, $n \in \mathbb{N}$. Задамо послідовність замкнених опуклих множин $M_{n} \subseteq V$ простої структури таку, що

$$
\left\{v \in V:\|v\|_{V} \leq \alpha_{n}\right\} \subseteq M_{n} \subseteq\left\{v \in V:\|v\|_{V} \leq \beta_{n}\right\} .
$$

У якості $M_{n}$ природно обирати опуклі багатогранники.

Опишемо метод розв'язання задачі (16), (17).

\section{Алгоритм 3. Метод лінеаризації з довірчою областю.}

1. Обираємо початкове наближення $u_{0} \in U$. Покладаємо $n=0$.

2. Знаходимо $y_{n} \in H-$ узагальнений розв'язок рівняння $\mathcal{L} y_{n}=F\left(u_{n}\right)$.

3. Знаходимо спряжений стан $p_{n} \in W_{+}-$розв'язок рівняння

$$
\mathcal{L}^{+} p_{n}=\Phi_{1}^{\prime}\left(y_{n}, u_{n}\right) .
$$

4. Знаходимо $\bar{u}_{n} \in U-$ розв'язок екстремальної задачі

$$
\left(\left(F^{\prime}\left(u_{n}\right)\right)^{*} p_{n}+\Phi_{2}^{\prime}\left(y_{n}, u_{n}\right), u-u_{n}\right)_{V} \rightarrow \varepsilon_{n}-\inf _{u \in U \cap\left(u_{n}+M_{n}\right)} .
$$

5. Покладаємо $u_{n+1}=\bar{u}_{n}, n:=n+1$ і переходимо на крок 2 . 
Зауваження 10. Алгоритм 3 належить до родини так званих методів 3 довірчою областю (Trust-Region Method) $[14,15]$. Зрозуміло, що в розглянутих алгоритмах допоміжна задача, яка розв'язується на кожній ітерації, $є$ локальною апроксимацією вихідної задачі чи умов оптимальності вихідної задачі. Ця апроксимація достовірна лише в досить малому околі поточного наближення. Тому природньо розв'язувати допоміжну задачу не на всій множині $U$, а саме на такому околі (який називають «довірчою областю»). Крім того, інтуїтивно ясно, що задача (18) є кращою апроксимацією вихідної задачі, ніж задача мінімізації вздовж довільного фіксованого напряму. Отже, на думку багатьох дослідників, можна очікувати, що незважаючи на високу ціну розв'язку допоміжної задачі (18), розв'язок цієї задачі забезпечить більший прогрес в мінімізації функціонала $J$, ніж одновимірний пошук, і алгоритм 3 може виявитись більш ефективним.

Для послідовності, що генерується описаним методом, має місце наступний результат про збіжність до множини стаціонарних керувань

$$
U^{*}=\left\{u^{*} \in U:\left(J^{\prime}\left(u^{*}\right), u-u^{*}\right)_{V} \geq 0, \forall u \in U\right\} .
$$

Теорема 7. Нехай:

$$
\varepsilon_{n}>0, k \cdot \alpha_{n} \geq \beta_{n} \geq \alpha_{n}>0, \sum_{n=0}^{\infty} \alpha_{n}=+\infty, \frac{\beta_{n}^{1+\gamma}}{\alpha_{n}} \rightarrow 0, \frac{\varepsilon_{n}}{\alpha_{n}} \rightarrow 0 ;
$$

множина $J\left(U^{*}\right)$ ніде не щільна. Тоді всі граничні точки послідовності $\left(u_{n}\right)$ утворюють компактну зв'язну підмножину в $U^{*}$, а числова послідовність $\left(J\left(u_{n}\right)\right)$ мае границяо.

Доведення. Знову використаємо загальну теорему про збіжність алгоритмів. За побудовою всі члени послідовності $\left(u_{n}\right)$ належать компакту $U$. Крім того, маємо $\left\|u_{n+1}-u_{n}\right\|_{V}=\left\|\bar{u}_{n}-u_{n}\right\|_{V} \leq \beta_{n} \rightarrow 0$ при $n \rightarrow \infty$.

Нехай $\left(u_{n_{k}}\right)$ - підпослідовність, що збігається до керування $u^{\prime} \notin U^{*}$. Покажемо, що існує таке $\delta_{0}>0$, що для всіх $k$ та $\delta \in\left(0, \delta_{0}\right]$ :

$$
\tau_{k}=\min _{n>n_{k}}\left\{n:\left\|u_{n}-u_{n_{k}}\right\|_{V}>\delta\right\}<+\infty .
$$

Від супротивного. Нехай для всіх $\delta_{0}>0$ існує $k_{0}=k_{0}\left(\delta_{0}\right)$ таке, що $\| u_{n}-$ $u_{n_{k_{0}}} \|_{V} \leq \delta_{0}$ для всіх $n>n_{k_{0}}$. Тоді з нерівностей

$$
\left\|u_{n}-u_{n_{k_{0}}}\right\|_{V} \leq \delta_{0},\left\|u_{n_{k}}-u_{n_{k_{0}}}\right\|_{V} \leq \delta_{0} \forall k>k_{0} \Rightarrow\left\|u^{\prime}-u_{n_{k_{0}}}\right\|_{V} \leq \delta_{0}
$$

випливає, що $u_{n} \in B_{2 \delta_{0}}\left(u^{\prime}\right)$ для всіх $n>n_{k_{0}}$.

Оцінимо приріст $J\left(u_{n+1}\right)-J\left(u_{n}\right)$. Маємо

$$
\begin{aligned}
J\left(u_{n+1}\right)-J\left(u_{n}\right) & \leq\left(J^{\prime}\left(u_{n}\right), u_{n+1}-u_{n}\right)_{V}+\frac{C_{1}}{\gamma+1}\left\|u_{n+1}-u_{n}\right\|_{V}^{\gamma+1} \leq \\
& \leq \min _{u \in U \cap\left(u_{n}+M_{n}\right)}\left(J^{\prime}\left(u_{n}\right), u-u_{n}\right)_{V}+\varepsilon_{n}+\frac{C_{1}}{\gamma+1} \beta_{n}^{\gamma+1} .
\end{aligned}
$$

Оскільки $u^{\prime} \notin U^{*}$, то існує число $\lambda>0$ таке, що

$$
\min _{u \in U}\left(J^{\prime}\left(u^{\prime}\right), u-u^{\prime}\right)_{V} \leq-2 \lambda<0 .
$$


Із неперервності функціонала $\xi \mapsto \min _{u \in U}\left(J^{\prime}(\xi), u-\xi\right)_{V}$ випливає існування $\delta>0$ такого, що для довільної точки $u^{\prime \prime} \in O_{\delta}\left(u^{\prime}\right) \bigcap U$ виконується нерівність

$$
\min _{u \in U}\left(J^{\prime}\left(u^{\prime \prime}\right), u-u^{\prime \prime}\right)_{V} \leq-\lambda<0 .
$$

Візьмемо $2 \delta_{0}<\delta$, тоді $u_{n} \in B_{2 \delta_{0}}\left(u^{\prime}\right) \subseteq O_{\delta}\left(u^{\prime}\right)$. Позначимо через $\overline{\bar{u}}_{n} \in U$ розв'язок задачі мінімізації $\left(J^{\prime}\left(u_{n}\right), u-u_{n}\right)_{V} \rightarrow \inf _{u \in U}$.

Нехай $\overline{\bar{u}}_{n} \in u_{n}+M_{n}$. Тоді з (19) випливає нерівність

$$
J\left(u_{n+1}\right)-J\left(u_{n}\right) \leq-\lambda+\varepsilon_{n}+\frac{C_{1}}{\gamma+1} \beta_{n}^{\gamma+1} .
$$

Існуе $n^{\prime}>n_{k_{0}}$ таке, що для всіх $n \geq n^{\prime}: J\left(u_{n+1}\right)-J\left(u_{n}\right) \leq-\frac{\lambda}{2}$.

Якщо $\overline{\bar{u}}_{n} \notin u_{n}+M_{n}$, то

$$
\begin{aligned}
J\left(u_{n+1}\right)-J\left(u_{n}\right) \leq\left(J^{\prime}\left(u_{n}\right),\right. & \left.\phi_{n}-u_{n}\right)_{V}+\varepsilon_{n}+\frac{C_{1}}{\gamma+1} \beta_{n}^{\gamma+1} \leq \\
& \leq \eta_{n}\left(J^{\prime}\left(u_{n}\right), \overline{\bar{u}}_{n}-u_{n}\right)_{V}+\varepsilon_{n}+\frac{C_{1}}{\gamma+1} \beta_{n}^{\gamma+1},
\end{aligned}
$$

де $\eta_{n}=\max \left\{\eta>0: \eta\left(\overline{\bar{u}}_{n}-u_{n}\right) \in M_{n}\right\}, \phi_{n}=u_{n}+\eta_{n}\left(\overline{\bar{u}}_{n}-u_{n}\right)$. У цьому випадку приріст оцінюється $J\left(u_{n+1}\right)-J\left(u_{n}\right) \leq-\eta_{n} \lambda+\varepsilon_{n}+\frac{C_{1}}{\gamma+1} \beta_{n}^{\gamma+1}$. Оскільки $1>\mu_{n} \geq \frac{\alpha_{n}}{\left\|\overline{\bar{u}}_{n}-u_{n}\right\|_{V}}$, то

$$
\begin{aligned}
J\left(u_{n+1}\right)-J\left(u_{n}\right) \leq & -\frac{\lambda}{\operatorname{diam}(U)} \alpha_{n}+\varepsilon_{n}+\frac{C_{1}}{\gamma+1} \beta_{n}^{\gamma+1}= \\
& =-\frac{\lambda}{\operatorname{diam}(U)} \alpha_{n}\left(1-\frac{\operatorname{diam}(U)}{\lambda}\left(\frac{\varepsilon_{n}}{\alpha_{n}}+\frac{C_{1}}{\gamma+1} \frac{\beta_{n}^{\gamma+1}}{\alpha_{n}}\right)\right) .
\end{aligned}
$$

Звідки випливає існування $n^{\prime \prime}>n_{k_{0}}$ такого, що для всіх $n \geq n^{\prime \prime}$

$$
J\left(u_{n+1}\right)-J\left(u_{n}\right) \leq-\frac{\lambda}{2 \cdot \operatorname{diam}(U)} \alpha_{n} \leq-\frac{\lambda}{2 \cdot k \cdot \operatorname{diam}(U)} \beta_{n} .
$$

Існує $n^{\prime \prime \prime} \in \mathbb{N}$ таке, що $\beta_{n}<\lambda / 2$ для всіх $n \geq n^{\prime \prime \prime}$. Отже,

$$
J\left(u_{n+1}\right)-J\left(u_{n}\right) \leq-\min \left\{1, \frac{\lambda}{2 \cdot k \cdot \operatorname{diam}(U)}\right\} \beta_{n},
$$

для $n>\tilde{n}=\max \left\{n^{\prime}, n^{\prime \prime}, n^{\prime \prime \prime}\right\}>n_{k_{0}}$.

Остаточно отримуємо

$$
J\left(u_{n}\right)-J\left(u_{n_{k}}\right) \leq-\min \left\{1, \frac{\lambda}{2 \cdot k \cdot \operatorname{diam}(U)}\right\} \sum_{p=n_{k}}^{n-1} \beta_{p}, n>n_{k}>\tilde{n} .
$$

Здійснивши граничний перехід в нерівності (20) при $n \rightarrow \infty$ та врахувавши $\sum_{p=1}^{\infty} \beta_{p}=+\infty$, отримаємо протиріччя з обмеженістю на компактній множині $U$ неперервного функціонала $J$.

Отже, існуе $\delta_{0}>0$ таке, що для всіх $k$ i $\delta \in\left(0, \delta_{0}\right]$ :

$$
\tau_{k}=\min _{n>n_{k}}\left\{n:\left\|u_{n}-u_{n_{k}}\right\|_{V}>\delta\right\}<+\infty .
$$


Але, обираючи достатньо мале $\delta_{0}>0$ та велике $k_{0}$, можна повторити доведення нерівності (20) для $n_{k} \leq n \leq \tau_{k}$. 3 іншого боку,

$$
\delta_{0}<\left\|u_{\tau_{k}}-u_{n_{k}}\right\|_{V} \leq \sum_{p=n_{k}}^{\tau_{k}-1}\left\|u_{p+1}-u_{p}\right\|_{V} \leq \sum_{p=n_{k}}^{\tau_{k}-1} \beta_{p} .
$$

Ураховуючи останню нерівність в (20), отримуємо

$$
J\left(u_{\tau_{k}}\right)<J\left(u_{n_{k}}\right)-\min \left\{1, \frac{\lambda}{2 \cdot k \cdot \operatorname{diam}(U)}\right\} \delta_{0} .
$$

Звідки маємо $\lim \sup _{k \rightarrow \infty} J\left(u_{\tau_{k}}\right)<\lim _{k \rightarrow \infty} J\left(u_{n_{k}}\right)$.

Покладемо $W=J$. Умови теореми про збіжність алгоритмів виконуються, а отже, послідовність $\left(u_{n}\right)$ має сформульовані властивості.

Перейдемо до дослідження поведінки алгоритму 3 без припущення, що точність $\varepsilon_{n}$ розв'язання допоміжної задачі прямує до нуля.

Припустимо, що

$$
\begin{gathered}
\left\{\varepsilon_{1}, \varepsilon_{2}, \ldots, \varepsilon_{n}, \ldots\right\} \subseteq[0, \bar{\varepsilon}], \quad \bar{\varepsilon}>0, \\
\beta_{n} \geq \alpha_{n} \geq \underline{\alpha}>0 \forall n \in \mathbb{N} \cup\{0\}, \\
\bar{\beta}=\limsup _{n \rightarrow \infty} \beta_{n} \in \mathbb{R} .
\end{gathered}
$$

Розглянемо множину

$$
U_{\bar{\varepsilon}, \underline{\alpha}, \bar{\beta}}^{*}=\left\{u^{*} \in U:\left(J^{\prime}\left(u^{*}\right), u-u^{*}\right)_{V} \geq-\operatorname{diam}(U)\left(\frac{\bar{\varepsilon}+C_{0} \bar{\beta}^{1+\gamma}}{\underline{\alpha}}\right), \forall u \in U\right\},
$$

де $C_{0}>0-$ константа з умови Гельдера на $U$ для похідної $J^{\prime}(\cdot)$.

Розглянемо породжену алгоритмом 3 послідовність $\left(u_{n}\right)$. Нехай $\left(u_{n_{k}}\right)-$ збіжна до керування $u^{\prime} \notin U_{\bar{\varepsilon}, \underline{\alpha}, \bar{\beta}}^{*}$ підпослідовність. Покажемо, що існує $\delta_{0}>0$ таке, що для всіх $k$ та $\delta \in\left(0, \delta_{0}\right] \tau_{k}=\min _{n>n_{k}}\left\{n:\left\|u_{n}-u_{n_{k}}\right\|_{V}>\delta\right\}<+\infty$. Від супротивного. Нехай для всіх $\delta_{0}>0$ існує $k_{0}=k_{0}\left(\delta_{0}\right)$ таке, що $\| u_{n}-$ $u_{n_{k_{0}}} \|_{V} \leq \delta_{0}$ для всіх $n>n_{k_{0}}$. Тоді з нерівності трикутника випливає, що $u_{n} \in B_{2 \delta_{0}}\left(u^{\prime}\right)$ для всіх $n>n_{k_{0}}$.

Оскільки $u^{\prime} \notin U_{\bar{\varepsilon}, \underline{\alpha}, \bar{\beta}}^{*}$, то існує число $\lambda>0$ таке, що

$$
\min _{u \in U}\left(J^{\prime}\left(u^{\prime}\right), u-u^{\prime}\right)_{V} \leq-\operatorname{diam}(U)\left(\frac{\bar{\varepsilon}+C_{0} \bar{\beta}^{1+\gamma}+2 \lambda}{\underline{\alpha}}\right) .
$$

Із неперервності функціонала $\xi \mapsto \min _{u \in U}\left(J^{\prime}(\xi), u-\xi\right)_{V}$ випливає існування $\delta>0$ і $\mu>0$ таких, що для довільної точки $u^{\prime \prime} \in O_{\delta}\left(u^{\prime}\right) \bigcap U$ виконується нерівність

$$
\min _{u \in U}\left(J^{\prime}\left(u^{\prime \prime}\right), u-u^{\prime \prime}\right)_{V} \leq-\operatorname{diam}(U)\left(\frac{\bar{\varepsilon}+C_{0}(\bar{\beta}+\mu)^{1+\gamma}+\lambda}{\underline{\alpha}}\right) .
$$


Візьмемо $2 \delta_{0}<\delta$, тоді $u_{n} \in B_{2 \delta_{0}}\left(u^{\prime}\right) \subseteq O_{\delta}\left(u^{\prime}\right)$. Маємо

$$
\begin{aligned}
J\left(u_{n+1}\right)-J\left(u_{n}\right) \leq & \left(J^{\prime}\left(u_{n}\right), u_{n+1}-u_{n}\right)_{V}+\frac{C_{0}}{\gamma+1}\left\|u_{n+1}-u_{n}\right\|_{V}^{\gamma+1} \leq \\
& \leq \min _{u \in U \cap\left(u_{n}+M_{n}\right)}\left(J^{\prime}\left(u_{n}\right), u-u_{n}\right)_{V}+\varepsilon_{n}+\frac{C_{0}}{\gamma+1} \beta_{n}^{\gamma+1} .
\end{aligned}
$$

Має місце нерівність

$$
\begin{aligned}
& \min _{u \in U \cap\left(u_{n}+M_{n}\right)}\left(J^{\prime}\left(u_{n}\right), u-u_{n}\right)_{V} \leq \min _{u \in U \cap B_{\alpha_{n}}\left(u_{n}\right)}\left(J^{\prime}\left(u_{n}\right), u-u_{n}\right)_{V} \leq \\
& \quad \leq \min _{u \in U \cap B_{\underline{\alpha}}\left(u_{n}\right)}\left(J^{\prime}\left(u_{n}\right), u-u_{n}\right)_{V} \leq \frac{\underline{\alpha}}{\operatorname{diam}(U)} \min _{u \in U}\left(J^{\prime}\left(u_{n}\right), u-u_{n}\right)_{V} .
\end{aligned}
$$

Отже, для $n>n_{k_{0}}$ справджується оцінка

$$
\begin{gathered}
J\left(u_{n+1}\right)-J\left(u_{n}\right) \leq \frac{\underline{\alpha}}{\operatorname{diam}(U)} \min _{u \in U}\left(J^{\prime}\left(u_{n}\right), u-u_{n}\right)_{V}+\varepsilon_{n}+\frac{C_{0}}{\gamma+1} \beta_{n}^{\gamma+1} \leq \\
\leq-\bar{\varepsilon}-C_{0}(\bar{\beta}+\mu)^{1+\gamma}-\lambda+\varepsilon_{n}+\frac{C_{0}}{\gamma+1} \beta_{n}^{\gamma+1} \leq \\
\leq-\lambda-C_{0}\left((\bar{\beta}+\mu)^{1+\gamma}-\beta_{n}^{\gamma+1}\right)-C_{0} \frac{\gamma}{\gamma+1} \beta_{n}^{\gamma+1} .
\end{gathered}
$$

Починаючи з деякого $n^{\prime}>n_{k_{0}}$ (не зменшуючи загальності, можна вважати, що $n^{\prime}=n_{k_{0}}$ ) виконується нерівність $\beta_{n}<\bar{\beta}+\mu$. Тому

$$
J\left(u_{n+1}\right)-J\left(u_{n}\right)<-\lambda-C_{0} \frac{\gamma}{\gamma+1} \beta_{n}^{\gamma+1}<-C_{0} \frac{\gamma}{\gamma+1} \beta_{n}^{\gamma+1} \leq-C_{0} \frac{\gamma}{\gamma+1} \underline{\alpha}^{\gamma+1} .
$$

Остаточно маємо

$$
J\left(u_{n}\right)-J\left(u_{n_{k}}\right)<-C_{0} \frac{\gamma}{\gamma+1}\left(n-1-n_{k}\right) \underline{\alpha}^{\gamma+1}, n>n_{k}>n_{k_{0}} .
$$

Здійснивши граничний перехід в нерівності (24) при $n \rightarrow \infty$, отримаємо протиріччя з обмеженістю на множині $U$ функціонала $J$. Отже, існує $\delta_{0}>0$ таке, що для всіх $k$ i $\delta \in\left(0, \delta_{0}\right]: \tau_{k}=\min _{n>n_{k}}\left\{n:\left\|u_{n}-u_{n_{k}}\right\|_{V}>\delta\right\}<+\infty$.

Але, обираючи достатньо мале $\delta_{0}>0$ та велике $k_{0}$, можна повторити доведення нерівності (5.2.19) для $n_{k} \leq n \leq \tau_{k}$. 3 іншого боку,

$$
\delta_{0}<\left\|u_{\tau_{k}}-u_{n_{k}}\right\|_{V} \leq \sum_{p=n_{k}}^{\tau_{k}-1}\left\|u_{p+1}-u_{p}\right\|_{V} \leq \sum_{p=n_{k}}^{\tau_{k}-1} \beta_{p} \leq\left(\tau_{k}-1-n_{k}\right) \sup _{p} \beta_{p} .
$$

Ураховуючи останню нерівність в (24), отримуємо

Звідки маємо

$$
J\left(u_{\tau_{k}}\right)-J\left(u_{n_{k}}\right)<-\delta_{0}\left(\frac{\gamma}{\gamma+1}\right) \frac{\underline{\alpha}^{\gamma+1} C_{0}}{\sup _{p} \beta_{p}} .
$$

$$
\limsup _{k \rightarrow \infty} J\left(u_{\tau_{k}}\right)<\lim _{k \rightarrow \infty} J\left(u_{n_{k}}\right) .
$$

Розглянемо тепер підпослідовність $\left(u_{n_{k}}\right)$ таку, що

$$
\lim _{k \rightarrow \infty} J\left(u_{n_{k}}\right)=\liminf _{n \rightarrow \infty} J\left(u_{n}\right) .
$$


Якщо припустити існування у обраної підпослідовності граничної точки $u^{\prime}$, що не належить множині $U_{\bar{\varepsilon}, \underline{\alpha}, \bar{\beta}}^{*}$, то, застосовуючи до $\left(u_{n_{k_{l}}}\right)\left(u_{n_{k_{l}}} \rightarrow u^{\prime}\right)$ нерівність (25), приходимо до протиріччя з (26).

Із наведених міркувань випливає

Теорема 8. Нехай виконуються умови (21), (22) i (23). Тоді згенерована алгоритмом 3 послідовність $\left(u_{n}\right)$ мае граничні точки, що належать множині $U_{\bar{\varepsilon}, \underline{\alpha}, \bar{\beta}}^{*}$.

\section{6. МОДЕЛІ АЛГОРИТМІВ УЗАГАЛЬНЕНОЇ ОПТИМІЗАЦІЇ ЛІНІЙНИХ СИСТЕМ 3 ПЕРЕДОПУКЛИМИ ДОПУСТИМИМИ МНОЖИНАМИ}

Ряд задач імпульсно-точкового керування системами з розподіленими параметрами та задач розміщення джерел фізичних полів породжують екстремальні задачі з так званими передопуклими обмеженнями.

Нехай $E-$ лінійний простір. Нагадаємо, що множину $M \subseteq E$ називаємо передопуклою, якщо існують такі опуклі множини $C_{0}, C_{1}, \ldots, C_{m}$, що

$$
M=C_{0} \backslash \bigcup_{k=1}^{m} C_{k} .
$$

Зауваження 11. Нагадаємо, що передопуклі множини, які можна представити у вигляді теоретико-множинної різниці двох опуклих множин, було введено у роботі [16] та використано у [17] для узагальнення методу проекції градієнта. У статті [18] введено більш широкий клас неопуклих множин, за якими збережено назву передопуклих, і розглянуто модифікацію методу умовного градієнта мінімізації гладких функцій на цих множинах.

Розглянемо задачу оптимального керування

$$
\begin{gathered}
J(u)=\Phi(y(u), u) \rightarrow \inf _{u \in U}, \\
\mathcal{L} y(u)=F(u), \quad u \in U,
\end{gathered}
$$

де $U$ - компактна множина допустимих керувань із скінченновимірного гільбертового простору керувань $V$. Припустимо, що множина $U$ має наступну структуру

$$
U=G_{0} \backslash \bigcup_{k=1}^{m} \operatorname{int} G_{k},
$$

де $G_{0}$ - опуклий компакт, $G_{1}, G_{2}, \ldots, G_{m}$ - замкнені опуклі множини, причому fr $G_{1}$, fr $G_{2}, \ldots$, fr $G_{m}$ - регулярні многовиди. Отже, $U$ - передопукла множина. Нехай $\operatorname{int} U \neq \emptyset$.

Відмітимо, що множини вигляду (29) природньо виникають в задачах точкового керування з «забороненими» зонами.

Введемо позначення $(k=1,2, \ldots, m)$ :

- $\Pi_{k}(u)$ - проекція точки $u \in U \backslash G_{k}$ на множину $G_{k}$;

- $n_{k}(u)$ - вектор зовнішньої нормалі до $G_{k}$ у точці $\Pi_{k}(u)$;

- $S_{k}(u)=\left\{v \in V:\left(n_{k}(u), v-\Pi_{k}(u)\right)_{V} \geq 0\right\}$ - дотичний півпростір до множини $G_{k}$ у $\Pi_{k}(u)$; 
- $P(u)=\bigcap_{k=1}^{m} P_{k}(u)$, де $P_{k}(u)=G_{0} \bigcap S_{k}(u)$.

Відмітимо, що $P(u)-$ непорожній опуклий компакт і $u \in P(u)$.

Будемо вважати, що про гладкість елементів екстремальної задачі (27), (28) виконані стандартні припущення цього розділу.

Для подальшого аналізу нам потрібна наступна необхідна умова мінімумy.

Лема 1. Нехай $\bar{u} \in U-$ локально оптимальний розв'язок задачі (27), (28). Toдi

$$
\min _{u \in P(\bar{u})}\left\langle\left(F^{\prime}(\bar{u})\right)^{*} \bar{p}+\Phi_{2}^{\prime}(y(\bar{u}), \bar{u}), u-\bar{u}\right\rangle_{V^{*}, V}=0,
$$

де $\bar{p}=p(\bar{u}) \in W_{+}-$розв'язок рівняння $\mathcal{L}^{+} p=\Phi_{1}^{\prime}(y(\bar{u}), \bar{u})$.

Доведення. Оскільки $\bar{u} \in P(\bar{u}) \subseteq U$, то керування $\bar{u}$ локально оптимальне на опуклій множині $P(\bar{u})$, а $(30)$ - класична необхідна умова локальної оптимальності для задачі

$$
\begin{gathered}
J(u)=\Phi(y(u), u) \rightarrow \inf , \\
\mathcal{L} y(u)=F(u), u \in P(\bar{u}) .
\end{gathered}
$$

Позначимо через $U^{*}$ множину керувань $u \in U$, для яких виконується необхідна умова мінімуму з леми 1.

Для розв'язання задачі керування (27), (28) розглянемо два ітераційні процеси.

Почнемо з модифікації методу умовного градієнта з роботи [18]. Джерело алгоритму - лема 1. Метод ідейно близький до методів з довірчою областю і полягає у розв'язанні на кожній ітерації допоміжних задач мінімізації лінійних форм на спеціально побудованих компактних опуклих множинах.

\section{Алгоритм 4. Метод лінеаризації для передопуклих задач.}

1. Обираємо початкове наближення $u_{0} \in U$. Покладаємо $n=0$.

2. Знаходимо $y_{n} \in H-$ узагальнений розв'язок рівняння $\mathcal{L} y_{n}=F\left(u_{n}\right)$.

3. Знаходимо спряжений стан $p_{n} \in W_{+}$- розв'язок рівняння

$$
\mathcal{L}^{+} p_{n}=\Phi_{1}^{\prime}\left(y_{n}, u_{n}\right) \text {. }
$$

4. Будуємо множину $P\left(u_{n}\right)=\cap_{k=1}^{m} P_{k}\left(u_{n}\right)$.

5. Знаходимо $\bar{u}_{n} \in U-$ розв'язок екстремальної задачі

$$
\left(\left(F^{\prime}\left(u_{n}\right)\right)^{*} p_{n}+\Phi_{2}^{\prime}\left(y_{n}, u_{n}\right), u-u_{n}\right)_{V} \rightarrow \varepsilon_{n}-\inf _{u \in P\left(u_{n}\right)} .
$$

6. Покладаємо $u_{n+1}=u_{n}+\rho_{n}\left(\bar{u}_{n}-u_{n}\right)$, де $\rho_{n} \in(0,1], n:=n+1$ i переходимо на крок 2.

Зауваження 12. Наближення $u_{n}$ завжди будуть лежати у компоненті зв'язності множини $U$, яка містить початкове наближення, що ускладнює при незв'язності множини $U$ знаходження глобального мінімуму $J(\cdot)$, навіть у випадку опуклості $J(\cdot)$ (наприклад, коли $\Phi(\cdot, \cdot)$ і $F(\cdot)$ лінійні). У 
даній ситуації слід запускати процес з початковими наближеннями 3 різних компонент зв'язності.

Зробимо додаткове припущення відносно множини $U$ :

$$
\forall u \in U: \operatorname{int} P(u) \neq \emptyset .
$$

Теорема 9. Нехай: виконуеться умова $(31) ; \rho_{n} \in(0,1], \rho_{n} \rightarrow 0, \sum_{n=0}^{\infty} \rho_{n}=$ $+\infty, \varepsilon_{n} \geq 0, \varepsilon_{n} \rightarrow 0$; функиіонал $J$ приймае на множині $U^{*}$ не більш ніэ зліченну кількість значень. Тоді всі граничні точки послідовності $\left(u_{n}\right)$ утворюють компактну зв'язну підмножсиу в $U^{*}$, а числова послідовність $\left(J\left(u_{n}\right)\right)$ мае границю.

Приділимо увагу моделі обчислень з не прямуючою при $n \rightarrow \infty$ до нуля похибкою $\varepsilon_{n}>0$. Будемо вважати, що $\varepsilon_{n} \in(0, \bar{\varepsilon}]$.

Введемо множину «майже стаціонарних» керувань

$$
U_{\bar{\varepsilon}}^{*}=\left\{u^{*} \in U: \min _{u \in P\left(u^{*}\right)}\left(J^{\prime}\left(u^{*}\right), u-u^{*}\right)_{V} \geq-\bar{\varepsilon}\right\} .
$$

Теорема 10. Нехай: виконуеться умова $(31) ; \rho_{n} \in(0,1], \rho_{n} \rightarrow 0, \sum_{n=0}^{\infty} \rho_{n}=$ $+\infty, \varepsilon_{n} \in(0, \bar{\varepsilon}]$. Тоді згенерована алгоритмом 4 послідовність $\left(u_{n}\right)$ мае граничні точки, що належать множині $U_{\bar{\varepsilon}}^{*}$.

Розглянемо тепер алгоритм, у якому на кожному кроці проводиться наближене проектування $\left(F^{\prime}\left(u_{n}\right)\right)^{*} p_{n}+\Phi_{2}^{\prime}\left(y_{n}, u_{n}\right)$ на допоміжну опуклу множину $P\left(u_{n}\right) \subseteq U$. Різні варіанти цієї процедури досліджувались в $[19,20]$.

Зауваження 13. Необхідна умова з леми 1 рівносильна рівності

$$
\bar{u}=\Pi_{P(\bar{u})}\left(\bar{u}-\left(F^{\prime}(\bar{u})\right)^{*} \bar{p}-\Phi_{2}^{\prime}(y(\bar{u}), \bar{u})\right),
$$

де $\Pi_{P(u)}(\cdot)$ - оператор проектування на множину $P(u), \bar{p}=p(\bar{u}) \in W_{+}-$ розв'язок рівняння $\mathcal{L}^{+} p=\Phi_{1}^{\prime}(y(\bar{u}), \bar{u})$.

\section{Алгоритм 5. Модифікований метод проекції градієнта.}

1. Обираємо початкове наближення $u_{0} \in U$. Покладаємо $n=0$.

2. Знаходимо $y_{n} \in H-$ узагальнений розв'язок рівняння $\mathcal{L} y_{n}=F\left(u_{n}\right)$.

3. Знаходимо спряжений стан $p_{n} \in W_{+}-$розв'язок рівняння

$$
\mathcal{L}^{+} p_{n}=\Phi_{1}^{\prime}\left(y_{n}, u_{n}\right) .
$$

4. Будуємо множину $P\left(u_{n}\right)=\cap_{k=1}^{m} P_{k}\left(u_{n}\right)$.

5. Знаходимо $\bar{u}_{n} \in U-$ розв'язок екстремальної задачі

$$
\left(\left(F^{\prime}\left(u_{n}\right)\right)^{*} p_{n}+\Phi_{2}^{\prime}\left(y_{n}, u_{n}\right), u-u_{n}\right)_{V}+\frac{1}{2}\left\|u-u_{n}\right\|_{V}^{2} \rightarrow \varepsilon_{n}-\inf _{u \in P\left(u_{n}\right)} .
$$

6. Покладаємо $u_{n+1}=u_{n}+\rho_{n}\left(\bar{u}_{n}-u_{n}\right)$, де $\rho_{n} \in(0,1], n:=n+1 \mathrm{i}$ переходимо на крок 2.

Теорема 11. Нехай: виконуеться умова $(31) ; \rho_{n} \in(0,1], \rho_{n} \rightarrow 0, \sum_{n=0}^{\infty} \rho_{n}=$ $+\infty, \varepsilon_{n} \geq 0, \varepsilon_{n} \rightarrow 0$; функціонал $J$ приймае на множині $U^{*}$ не більш ніж зліченну кількість значень. Тоді всі граничні точки породжсеної алгоритмом 5 послідовності $\left(u_{n}\right)$ утворюють компактну зв'язну підмножину в $U^{*}$, а числова послідовність $\left(J\left(u_{n}\right)\right)$ мае границю. 


\section{ЗАКЛЮЧНІ ЗАУВАЖЕННЯ}

У роботі розглянуті задачі оптимізації лінійних розподілених систем 3 узагальненим керуванням та методи першого порядку їх розв'язання. Основну увагу приділено обгрунтовуванню збіжності методів. Припускається, що оператор, який описує модель, задовольняє апріорні оцінки в негативних нормах. Для задач керування з опуклими та передопуклими допустимими множинами доведено збіжність аналогів алгоритмів лінеаризації, проекції градієнта та довірчої області із похибками в ітераційних підзадачах.

Робота виконана за фінансової підтримки МОН України (проєкт «Математичне моделювання та оптимізація динамічних систем для оборони, екології та медицини», 0219U008403, 2019-2021 рр.) та НАН України (проєкт «Нові методи дослідження коректності та розв'язання задач дискретної оптимізації, варіаційних нерівностей та їх застосування», 0119U101608, 2019-2020 pp.).

\section{ЛiтературА}

1. Ляшко С. И. Импульсное оптимальное управление линейными системами с распределенными параметрами. ДАН СССР. 1984. Т. 276. № 2. С. 285-287.

2. Ляшко С. И. Оптимизация линейных систем с обобщенным воздействием. Обозрение прикладной и промыиленной математики. 1995. № 1. С. 70-77.

3. Lyashko S. I. Generalized optimal control of linear systems with distributed parameters. Boston-Dordrecht-London: Kluwer Academic Publishers, 2002.466 p.

4. Ляшко С. И., Семенов В. В. Об управляемости линейных распределенных систем в классах обобщенных воздействий. Кибернетика и системный анализ. 2001. № 1. C. $18-41$.

5. Ляшко С. І., Номіровський Д. А., Семенов В. В. Дослідження лінійних розподілених систем з узагальненим керуванням. ЖКрнал обчислювальної та прикладної математики. 2004. № 2 (91). С. 31-45.

6. Березанский Ю. М. Разложение по собственным функциям самосопряженных операторов. Киев: Наук. думка, 1965. 800 с.

7. Сеа Ж. Оптимизация. Теория и алгоритмы. Москва: Мир, 1973. 244 с.

8. Ермольев Ю. М. Методы стохастического программирования. Москва: Наука, 1976. 239 c.

9. Васильев Ф. П. Численные методы решения экстремальных задач. М.: Наука, 1988. $549 \mathrm{c}$.

10. Карманов В. Г. Математическоепрограммирование. Москва:Наука, 1986. 286 с.

11. Измаилов А. Ф., Солодов М. В. Численные методы оптимизации. Москва: ФИЗМАТЛИТ, 2005.

12. Нурминский Е. А. Численные методы решения детерминированных и стохастических минимаксных задач. Киев: Наук. думка, 1979. 159 с.

13. Михалевич В. С., Гупал А. М., Норкин В. И. Методы невыпуклой оптимизации. Москва: Наука, 1987. 279 с.

14. Conn R., Gould N. I. M., Sartenaer A., Toint P. L. Global convergence of a class of trust region algorithms for optimization using inexact projections onto convex constraints. SIAM J. Optim. 1993. Vol. 3. P. 164-221. 
15. Heinkenschloss M., Vicente L. N. Analysis of Inexact Trust-Region SQP Algorithms. SIAM J. Optim. 2002 Vol. 12. No. 2. P. 283-302.

16. Заботин В. И., Полонский Ю. А. Предвыпуклые множества, отображения и их приложения к экстремальным задачам. Кибернетика. 1981. № 5. С. 71-74.

17. Заботин В. И., Черняев Ю. А. Обобщение метода проекции градиента на экстре-мальные задачи с предвыпуклыми ограничениями. Журнал вычислительной математики и математической физики. 2001. Т. 41. № 3. С. 367373.

18. Семенов В. В. Модификация метода условного градиента для решения экстремальных задач на одном классе невыпуклых множеств. Журнал обчислювальної та прикладної математики. 2002. № 1 (87). С. 72-76.

19. Лисюк В. М., Семенов В. В., Семенов Вік. В., Сорока Ю. Б. Метод послідовної квадратичної оптимізації розв'язання задач на передопуклих множинах. Вісник Київсъкого університету. Серія: фiз.-мат. науки. 2006. Вип. 4. С. 186-190.

20. Клюшин Д. А., Ляшко Н. И., Номировский Д. А., Семенов В. В. Об одном методе точечной оптимизации. Журнал обчислювалъної та прикладної математики. 2005. № 2 (93). С. 79-84.

Надійшла: 12.10.2020 / Прийнята: 30.11.2020

\title{
МЕТОДЫ ПЕРВОГО ПОРЯДКА ДЛЯ ЗАДАЧ ОБОБЩЕННОГО ОПТИМАЛЬНОГО УПРАВЛЕНИЯ СИСТЕМАМИ С РАСПРЕДЕЛЕННЫМИ ПАРАМЕТРАМЫ
}

\author{
С. В. ДЕнисов, В. В. Семенов
}

Факультет компьютерных наук и кибернетики, Киевский национальный университет имени Тараса Шевченко, Киев, Украина, E-mail: \{sireukr, volodya.semenov\}@gmail.com

АннотАция. Рассматриваются задачи оптимизации линейных распределенных систем с обобщенным управлением и методы первого порядка их решения. Основное внимание уделено обоснованию сходимости методов. Предполагается, что оператор, описывающий модель, удовлетворяет априорные оценки в негативных нормах. Для задач управления с выпуклыми и предвыпуклыми допустимыми множествами доказана сходимость нескольких алгоритмов первого порядка с погрешностями в итерационных подзадачах.

КлЮчЕВЫЕ СЛовА: оптимальное управление, система с распределенными параметрами, обобщенное управление, метод первого порядка, сходимость. 